\title{
Metal ion-responsive nanocarrier derived from phosphonated calix[4]arenes for delivering dauricine specifically to sites of brain injury in a mouse model of intracerebral hemorrhage
}

Mingxin Li ${ }^{1,2}$, Guohao Liu ${ }^{1,5}$, Kaixuan Wang ${ }^{1,2}$, Lingfeng Wang ${ }^{1,2}$, Xiang Fu ${ }^{6}$, Lee Yong Lim ${ }^{4}$, Wei Chen ${ }^{1,2^{*}}$ and Jingxin $\mathrm{Mo}^{1,3^{*}}$ (D)

\begin{abstract}
Primary intracerebral hemorrhage $(\mathrm{ICH})$ is a leading cause of long-term disability and death worldwide. Drug delivery vehicles to treat $\mathrm{ICH}$ are less than satisfactory because of their short circulation lives, lack of specific targeting to the hemorrhagic site, and poor control of drug release. To exploit the fact that metal ions such as $\mathrm{Fe}^{2+}$ are more abundant in peri-hematomal tissue than in healthy tissue because of red blood cell lysis, we developed a metal ion-responsive nanocarrier based on a phosphonated calix[4]arene derivative in order to deliver the neuroprotective agent dauricine (DRC) specifically to sites of primary and secondary brain injury. The potential of the dauricine-loaded nanocarriers for $\mathrm{ICH}$ therapy was systematically evaluated in vitro and in mouse models of autologous whole blood double infusion. The nanocarriers significantly reduced brain water content, restored blood-brain barrier integrity and attenuated neurological deficits by inhibiting the activation of glial cells, infiltration by neutrophils as well as production of proinflammatory factors (IL-1 $\beta$, IL-6, TNF- $a$ ) and matrix-metalloprotease-9. These results suggest that our dauricine-loaded nanocarriers can improve neurological outcomes in an animal model of $\mathrm{ICH}$ by reducing inflammatory injury and inhibiting apoptosis and ferroptosis.
\end{abstract}

Keywords: Intracerebral hemorrhage, Dauricine, Phosphonated calix[4]arene derivative, Targeted delivery, Apoptosis, Ferroptosis

\section{Introduction}

Primary intracerebral hemorrhage $(\mathrm{ICH})$ is the most devastating type of stroke [1]. It affects 2 million people worldwide each year and is associated with high disability and mortality rates, which have not changed substantially for decades. Individuals who suffer ICH have poor prognosis mainly because of secondary brain injuries after the stroke, including hematoma toxicity, oxidative

*Correspondence: daicw1104@163.com; Jingxin.mo@hotmail.com ${ }^{1}$ Clinical Research Center for Neurological Diseases of Guangxi Province, The Affiliated Hospital of Guilin Medical University, Guilin 541001, China Full list of author information is available at the end of the article stress and inflammatory injury [2]. Much secondary injury arises from neuroinflammation: hematoma activates glial cells, which disrupt the blood-brain barrier, allowing infiltration by peripheral inflammatory cells that produce abundant cytokines, which in turn stimulate neuronal apoptosis and impair neurological function [3, $4]$. Another major cause of secondary injury is ferroptosis due to the abundant free iron released from lysed erythrocytes in stroke-injured brain tissue [5,6]. The complexity of ICH helps explain why no specific treatment exists, despite the burden it places on health systems worldwide.

A neuroprotective agent that may help mitigate secondary stroke injury is the isoquinoline alkaloid dauricine 
(DRC), isolated from the Chinese herbal medicine Rhizoma Menispermi (Scheme 1) [7]. DRC can protect the brain from ischemic damage by up-regulating $\mathrm{Bcl}-2$ and down-regulating Bax expression, thereby inhibiting neuronal apoptosis, as well as by stimulating IRE-1/XBP-1 signaling and down-regulating caspase-3, which relieves

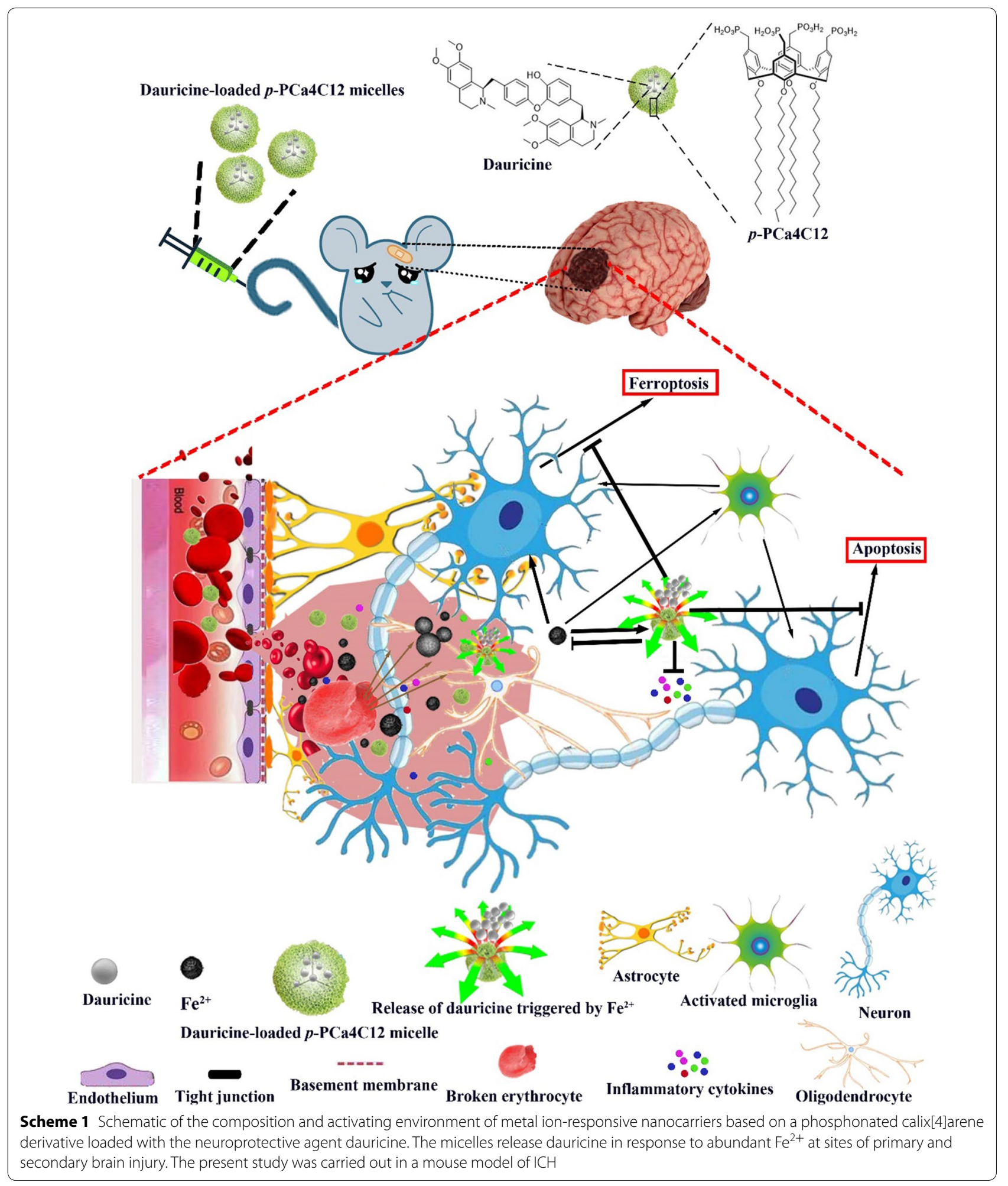


endoplasmic reticulum stress [8]. However, DRC on its own shows poor oral absorption, high rate of metabolism and rapid systemic elimination, which means its actual concentration at hemorrhagic sites is very low [9].

We hypothesized that we could couple DRC with an appropriate carrier that would be responsive to metal ions such as $\mathrm{Fe}^{2+}$ that are more abundant at hematoma sites than in normal tissue. As a drug delivery system, we focused on the calixarene macrocyle and its derivatives, which have antiviral, antibacterial, antifungal, antitubercular, anticancer, and anti-oxidant activities [10-13]. We have shown that a $p$-methylenebisphosphonic calix[4] arene derivative can assemble into micelles to form host-guest complexes with small molecules in the cavity, such as the drug carboplatin [14, 15]. Furthermore, calixarene derivatives can form host-guest complexes with free metal ions such as $\mathrm{Cu}^{2+}$ and $\mathrm{Fe}^{2+}$, in some cases with metal selectivity [16-19]. We envisioned that using calixarene derivatives loaded with DRC could synergistically treat $\mathrm{ICH}$ by binding selectively to metal ions and by releasing DRC selectively at hemorrhagic sites. In the present study, we designed $O$-dodecyl $p$-methylenebisphosphonic calix[4]arene micelles containing DRC (hereafter referred to as DPM) and explored their antiinflammatory and neuroprotective effects in a mouse model of ICH (Scheme 1).

\section{Materials and methods Reagents}

All reagents, solvents, chemicals and plastic cell culture supplies were obtained from Sigma-Aldrich (St. Louis, MO, USA) or Fisher (Pittsburgh, PA, USA) unless otherwise mentioned. Annexin V-FITC/PI Apoptosis Kits were purchased from Lianke Technology (Hangzhou, China). Matrigel ${ }^{\circledR}$ Basement Membrane Matrix was obtained from Corning (NY, USA). Ammonium ferrous sulfate was ordered from Macklin (Shanghai, China). ROS Assay Kit was obtained from Beyotime (Shanghai, China). DRC was obtained from Aladdin Chemical Reagent Co., Ltd (Shanghai, China). Paraformaldehyde (4\%) was purchased from Guangzhou Ruishu Biotechnology Co., Ltd (Guangzhou, China). Ultrapure deionized water was obtained from a Millipore system (resistivity, $18.2 \mathrm{M} \Omega \mathrm{cm}$ ). Phosphonato calixarene $(p-\mathrm{PCa} 4 \mathrm{C} 12$, purity $>95 \%$ ) was synthesized in our laboratory according to published methods with some minor modifications. Its chemical structure was confirmed by ${ }^{1} \mathrm{H}-\mathrm{NMR}$ (Varian Mercury 400, USA; Additional file 1: Figure S1).

\section{Cells}

Human SH-SY5Y neuroblastoma cell lines (Cell Resource Centre, Guilin Medical University, China) were cultured in complete medium (Dulbecco's modified Eagle's medium, DMEM) supplemented with $10 \%$ fetal bovine serum (FBS; Gibco, Invitrogen, Shanghai, China) Cultures were maintained in a humidified $5 \% \mathrm{CO}_{2}$ atmosphere.

\section{Animals and groups}

Animal protocols were approved by the Department of Laboratory Animal Research at Guilin Medical University (License No. YXLL-2017-167) in compliance with the Principles of Laboratory Animal Care (People's Republic of China). Adult male C57BL/6 mice weighing 20 to $28 \mathrm{~g}$ were used in this study. Mice were maintained at a constant ambient temperature $\left(22 \pm 1{ }^{\circ} \mathrm{C}\right)$ on a 12-h light/ dark cycle. Mice were randomly divided into the following five groups based on random numbers generated using SPSS (IBM, Chicago, IL, USA). The sham group $(\mathrm{n}=24$, of which 24 survived) was subjected to mock surgery (craniotomy without blood infusion) and treated with $0.1 \mathrm{~mL} 0.9 \%$ saline. The vehicle group $(n=26$, of which 21 survived) was subjected to ICH surgery, then treated with $0.9 \%$ saline. The DRC group $(n=24$, of which 21 survived) was subjected to ICH surgery, then immediately treated with $10 \mathrm{mg} / \mathrm{kg}$ DRC (Sigma, purity $\geq 95 \%$, Fig. 1) via tail vain injection. The drug was dissolved in $0.05 \mathrm{~mL} \mathrm{20.0 \%} \mathrm{(v/v)} \mathrm{HCl}$, then neutralized with $\mathrm{NaOH}$ (Sigma, USA, purity $\geq 99.0 \%)$. The PM group $(n=24$, of which 20 survived) was subjected to ICH surgery, then treated with $90 \mathrm{mg} / \mathrm{kg}$ empty $p$-PCa4C12 micelles (PM). The DPM group ( $\mathrm{n}=24$, of which 22 survived) was subjected to ICH surgery, then treated with $100 \mathrm{mg} / \mathrm{kg}$ dauricine-loaded $p$-PCa4C12 micelles (DPM, equivalent to $10.1 \mathrm{mg} / \mathrm{kg}$ DRC).

\section{Fabrication of PM and DPM}

DPM were fabricated by a thin-film hydration method. In brief, $100 \mathrm{mg}$ of $p$-PCa4C12 and $30 \mathrm{mg}$ of DRC were dissolved in $50 \mathrm{~mL}$ of chloroform in a $150-\mathrm{mL}$ round-bottom flask. The flask was attached to a rotary evaporator (BUCHI, Switzerland) to get rid of chloroform overnight in a $37^{\circ} \mathrm{C}$ water bath. The resultant thin film was rehydrated at $37{ }^{\circ} \mathrm{C}$ for half an hour in $20 \mathrm{~mL}$ of deionized water and then sonicated (probe sonicator, 50\% strength; Q700 of QSonica ${ }^{\circledR}$, Newtown, CT, USA) for $5 \mathrm{~min}$ to form DPM. Insoluble material was eliminated using a $0.22-\mu \mathrm{m}$ filter (Millipore filter), and the filtered DPM were freeze-dried and stored at $-20^{\circ} \mathrm{C}$ for further experiments. The same procedure but without DRC was used to prepare PM.

\section{Physical characterization of micelle formulations}

Particle size, polydispersity index (PDI), and zeta potential of DPM and PM were investigated using dynamic light scattering (Malvern Nano ZS90 laser 

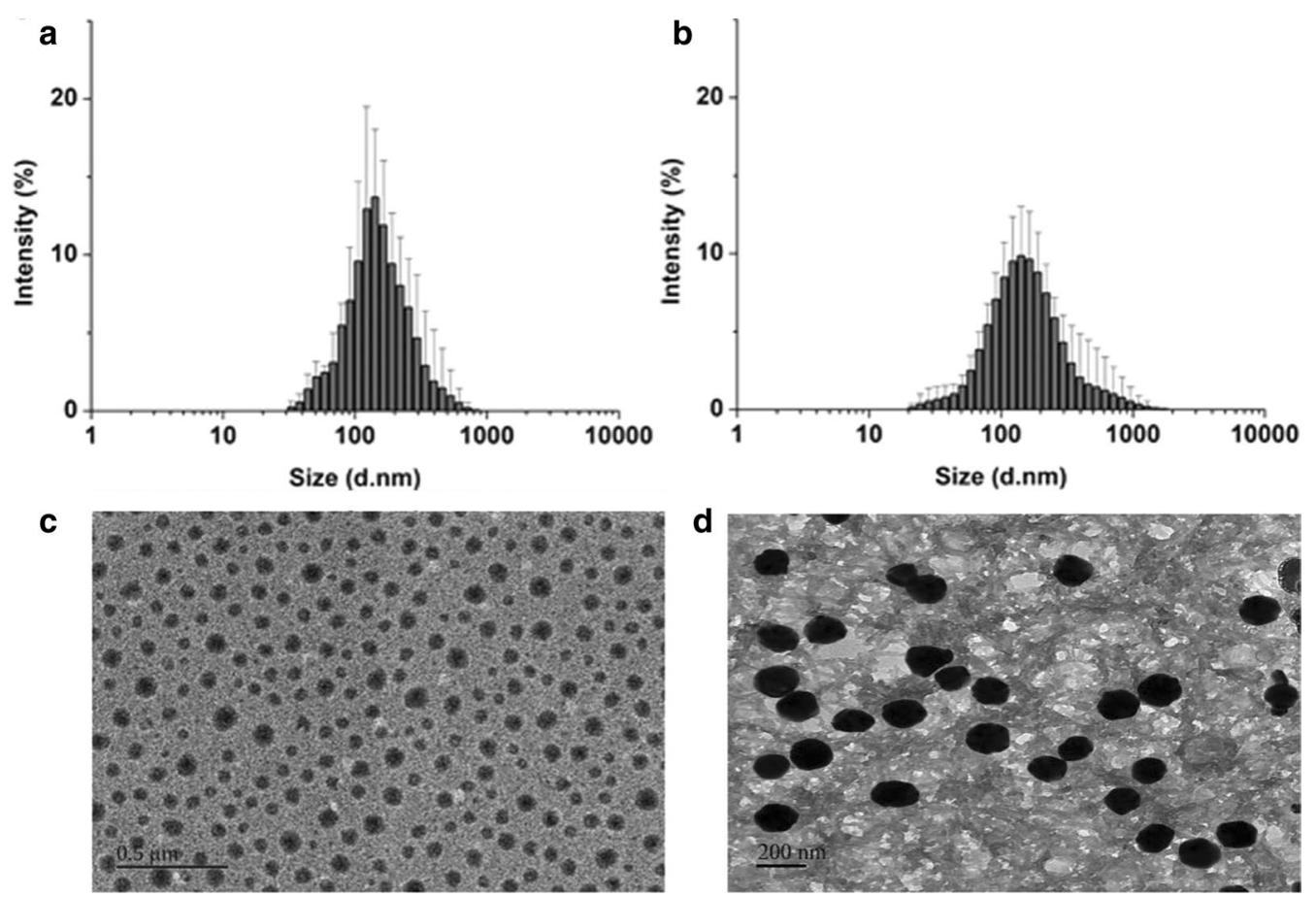

Fig. 1 Characterization of size and morphology of blank p-PCa4C12 micelles (PM) and dauricine-loaded p-PCa4C12 micelles (DPM). Dynamic light scattering was used to analyze the size distribution of $\mathbf{a}$ PM and $\mathbf{b}$ DPM, while transmission electron microscopy was used to examine morphology of c PM, Scale bar, $0.5 \mu \mathrm{m}$ and $\mathbf{d}$ DPM, Scale bar, $200 \mathrm{~nm} . \mathrm{n}=5$

particle size analyzer, Malvern, UK). Morphology of PM and DPM was analyzed using transmission electron microscopy (JEOL, Japan).

\section{In vitro release profiles and stability of DPM in different} $\mathrm{Fe}^{2+}$ concentrations

Lyophilized DPM was precisely weighed and dispersed in release medium [phosphate-buffered saline (PBS, $\mathrm{pH}$ 7.4) with or without $5 \mathrm{mM}$ ammonium ferrous sulfate] to obtain a final DRC concentration of $2 \mu \mathrm{M}$. DPM solution $(5 \mathrm{~mL})$ was placed into a sealed dialysis bag (Spectrum Laboratories, Rancho Dominguez, CA, USA) with a molecular weight cut-off of $1000 \mathrm{Da}$, then transferred to $95 \mathrm{~mL}$ of phosphate-buffered saline (PBS, pH 7.4) containing 0.5\% Tween 80 with or without $5 \mathrm{mM} \mathrm{Fe}^{2+}$ (ammonium ferrous sulfate). The system was incubated at $37 \pm 0.5{ }^{\circ} \mathrm{C}$ with stirring at $100 \mathrm{rpm}$. At specific intervals, the medium containing the dialysis bag was sampled $(0.5 \mathrm{~mL})$, then fresh $0.5 \mathrm{~mL}$ release medium was replaced, and DRC was assayed using HPLC (Shimadzu LC-20A) as described in Supplementary Information. All procedures, including incubation and HPLC detection, were carried out under minimal light conditions.

\section{Neuroprotective effects of DPM}

SH-SY5Y cells were seeded in 96-well plates in $100 \mu \mathrm{L}$ of DMEM medium $\left(1 \times 10^{4}\right.$ cells/well $)$ and incubated for $24 \mathrm{~h}$. Free DRC, PM or DPM was added to wells at a series of concentrations $(0.01 \mu \mathrm{M}-1 \mu \mathrm{M})$, with all wells except for negative control group also receiving ammonium ferrous sulfate to a final concentration of $5 \mathrm{mM}$. Cells were incubated another $24 \mathrm{~h}$ at $37^{\circ} \mathrm{C}$ and cell viability was tested using the CCK- 8 assay.

\section{Assay of intracellular reactive oxygen species (ROS)}

Intracellular ROS generation in SH-SY5Y cells was detected using the ROS Assay Kit. Typically, SH-SY5Y cells were seeded into a $35-\mathrm{mm}$ glass-bottom culture dish (NEST, China) at a density of $1 \times 10^{6}$ cells/well and cultured overnight in $2 \mathrm{~mL}$ DMEM medium with 10\% FBS at $37{ }^{\circ} \mathrm{C}$. The medium was replaced with fresh DMEM and $10 \% \mathrm{FBS}$ with or without $\mathrm{Fe}^{2+}$ at a final concentration of $5 \mathrm{mM}$, followed by addition of $1 \mu \mathrm{M}$ DRC, $5 \mu \mathrm{M}$ PM or DPM to a final DRC concentration of $1 \mu \mathrm{M}$. Negative control cultures were not exposed to $\mathrm{Fe}^{2+}$, free DRC or micelles. The vehicle group was treated with $\mathrm{Fe}^{2+}$ and then $0.1 \mathrm{~mL}$ DMSO. After 12-h incubation, the medium was replaced with fresh DMEM $(2 \mathrm{~mL})$ containing ROS reagent $(10 \mu \mathrm{M})$, then cells were incubated 
another $20 \mathrm{~min}$, washed three times with ice-cold PBS and imaged and semi-quantified with fluorescent confocal microscopy (Leica TCS SP5, Germany). The extent of ROS-mediated decomposition of 2'7'-Dichlorodihydrofluorescein diacetate (DCFH-DA) into dichlorofluorescein (DCF) was measured at an excitation wavelength of $488 \mathrm{~nm}$ and emission wavelengths of 500 to $540 \mathrm{~nm}$. Three fields of view were randomly chosen for each group before Image-Pro Plus 5.1 software (Media Cybernetics Inc., Rockville, MD, USA) was used for quantitative analysis of ROS (area $\times$ intensity).

\section{Apoptosis detection based on Annexin V / propidium iodide staining}

SH-SY5Y cells were cultured for $12 \mathrm{~h}$ in the presence of $5 \mathrm{mM} \mathrm{Fe}^{2+}$ (to induce apoptosis) as well as $1 \mu \mathrm{M}$ DRC, $5 \mu \mathrm{M}$ PM or DPM (final DRC concentration, $1 \mu \mathrm{M}$ ). Then the medium was replaced with fresh DMEM and the cells were incubated another $24 \mathrm{~h}$. Cells were trypsinized, collected by centrifugation at $300 \mathrm{~g}$ for $5 \mathrm{~min}$, rinsed twice with PBS and resuspended in $500 \mu \mathrm{L}$ of binding buffer. The cells were mixed with $5 \mu \mathrm{L}$ of Annexin V-FITC and propidium iodide (PI), then the suspension was mixed and incubated in the dark for $15 \mathrm{~min}$. Cell apoptosis was analyzed by flow cytometry (Becton Dickinson, Franklin Lake, NJ, USA).

\section{Mouse model of autologous blood ICH}

ICH was induced using the autologous whole blood double infusion model ( $30 \mu \mathrm{L}$ total infusion). Mice (5-week-old male; Orient Bio, Hunan, China) were anesthetized with continuous isoflurane ( $4 \%$ induction, 1.5-2\% maintenance) and immobilized on a stereotactic frame (Stoelting, Wood Dale, IL, USA). After making a small midline sagittal incision in the skin overlying the skull, a craniotomy was performed $0.5 \mathrm{~mm}$ anterior and $2.4 \mathrm{~mm}$ right relative to the bregma [20, 21] (Additional file 1: Figure S2). Autologous blood was collected onto a sterile surface by needle prick of the tail artery after first cleaning the area with 70\% ethanol and gently warming the tail for 2 min with a heat lamp. Blood was immediately drawn into PE-20 tubing (Instech, Plymouth Meeting, PA) connected on one side to a $50 \mathrm{~mL}$ syringe with a 26-gauge luer tip needle (Hamilton Company, Reno, NV, USA) located within an automated injector, and the other side to a 26-gauge needle with the beveled end inserted into the tubing [21]. The blunt end of this needle was inserted $3.9 \mathrm{~mm}$ ventral from the skull surface, relocated to $3.6 \mathrm{~mm}$, and left in place for $7 \mathrm{~min}$. After the waiting period, $10 \mu \mathrm{L}$ of blood was infused, followed by an additional 5 min waiting period prior to a second infusion of $20 \mu \mathrm{L}$ [22]. All injections were performed at $1.0 \mu \mathrm{L} / \mathrm{min}$ using an automated injector (Stoelting, Wood Dale, IL,
USA). The needle was left in place for $10 \mathrm{~min}$ after the second infusion, then slowly removed over a 25-min period. The sham model was set up by performing craniotomy without blood infusion.

\section{Behavior testing}

Two trained investigators (Lingfeng Wang and Kaixuan Wang) who were blinded to group allocations determined the modified neurological severity score (mNSS) at $24 \mathrm{~h}$ after ICH. This assessment of neurological deficit evaluates abnormal movements as well as motor, sensory, and reflex deficits, which are scored on an 18-point neurological deficit scale. Maximal deficit score was 18, with higher scores indicating worse deficit. Eight animals were randomly chosen from each group for evaluation.

\section{Brain water content measurement}

Brain water content was measured after ICH as previously described [23]. Brains $(\mathrm{n}=8)$ were harvested $24 \mathrm{~h}$ after $\mathrm{ICH}$, and 4-mm-thick coronal brain samples were collected from $2 \mathrm{~mm}$ anterior and $2 \mathrm{~mm}$ posterior to the injection site. The brains were divided into 2 parts: ipsilateral and contralateral hemisphere. Brain samples were immediately weighed to obtain the wet weight and then dried at $70{ }^{\circ} \mathrm{C}$ for $48 \mathrm{~h}$ to obtain the dry weight. Brain water content (\%) was calculated as (wet weight-dry weight)/wet weight $\times 100 \%$.

\section{Assessment of blood-brain barrier permeability}

The Evans blue extravasation test was performed to examine the permeability of the blood-brain barrier as previously described [24]. Evans blue $(2 \%, 4 \mathrm{~mL} / \mathrm{kg}$, Sigma, USA) was injected into the tail vein of the mice at $24 \mathrm{~h}$ after ICH. After $2 \mathrm{~h}$, mice $(\mathrm{n}=5-6)$ were euthanized and perfused with $250 \mathrm{~mL}$ saline. Brain hemispheres were quickly removed and weighed. Each sample was incubated in trichloroacetic acid, homogenized and centrifuged. The supernatant was diluted with ethanol. The absorbance of the supernatant solution at $620 \mathrm{~nm}$ was measured using a spectrophotometer (Thermo Fisher Scientific, USA) and quantified based on a standard curve.

\section{Ex vivo imaging of micelle accumulation in the brain}

For ex vivo imaging studies, after the $\mathrm{ICH}$ mouse model was set up, groups of C57BL/6 mice were injected with $1 \mu \mathrm{g} / \mathrm{g}$ of the near-infrared dye DiR $\left(1,1^{\prime}\right.$-Dioctadecyl$3,3,3^{\prime}, 3^{\prime}$-Tetramethylindotricarbocyanine Iodide) on its own or loaded into PM (DiPM). Stock solutions were prepared as $1 \mathrm{mg}$ DiR in $100 \mathrm{~mL} 0.9 \%$ saline or an equivalent amount of DiPM in $100 \mathrm{~mL} 0.9 \%$ saline. At 12 and $24 \mathrm{~h}$ of injection, organs were then excised from mice and imaged with an IVIS Spectrum imaging system (Caliper, 
USA) with excitation at $748 \mathrm{~nm}$ and absorption at $780 \mathrm{~nm}$ in order to capture the fluorescence emitted by DiR.

\section{MRI image acquisition}

Data were obtained on a SIEMENS Verio 3.0 Tesla MRI scanner (Siemens Healthineers, Erlangen, Germany) using a mouse magnetic resonance coil (WanKang, Wuxi, China). All images were analyzed by experienced radiologists who were blinded to the animal treatment. The imaging protocol for all mice included a T2 fast spinecho. The field of view was $20 \times 20 \mathrm{~mm}$, and the matrix was $256 \times 256 \mathrm{~mm}$. Seven coronal slices $(1 \mathrm{~mm}$ thick) were acquired from the frontal pole to the brain stem, and the images were saved as images of $256 \times 256$ pixels.

\section{Histology and quantification}

Histology and quantification were conducted as previously described [25] with minor modifications. Briefly, 10 sets of 16 sections equally distributed throughout the hematoma and anteroposterior brain regions were processed. The tissue blocks were cut into 4- $\mu \mathrm{m}$ sections and stained with hematoxylin and eosin to assess pathological changes in the brain. Cresyl violet staining was used to assess lesion and hematoma volume, tissue injury, percent ipsilateral hemispheric enlargement, and ventricular volume. Perls' iron staining was used to evaluate iron deposition. Photomicrographs were acquired using a light microscope (Nikon ECLIPSE 80i, Nikon, Tokyo, Japan).

\section{Western blot analysis}

Brain samples were obtained at $24 \mathrm{~h}$ after $\mathrm{ICH}$. Protein from perihematomal tissue $(n=4-6)$ was extracted and analyzed according to our previous study [26]. In brief, the brain sample was homogenized and centrifuged. An equal amount of protein from each sample was mixed with loading buffer, denatured, separated on an $8-12 \%$ $(\mathrm{v} / \mathrm{v})$ SDS gel, and transferred to nitrocellulose membranes. Membranes were blocked with 5\% nonfat dry milk and incubated with primary antibody against the following proteins: GPX4 (ab41787, Abcam), caspase-3 (ab90437), MMP9 (sc-6841, Santa Cruz Biotechnology), ZO-1 (sc-8147), $\beta$-actin (sc-47778), Bcl-2 (sc-783), and $\mathrm{Bax}$ (sc-6236). Then membranes were treated with horseradish peroxidase-conjugated anti-rabbit/mouse IgG secondary antibody, detected with a chemiluminescence substrate (Thermo Scientific) and visualized using the ChemiDoc ${ }^{\mathrm{TM}}$ MP Imaging System (Bio-Rad). The density of blot bands was quantified using ImageJ software $(\mathrm{NIH}$, USA).

\section{Elisa}

Perihematomal brain tissue $(n=5-6)$ was collected at $24 \mathrm{~h}$ after $\mathrm{ICH}$, homogenized and centrifuged. The supernatant was assayed for IL-1 $\beta$, IL- 6 and TNF- $\alpha$ using enzyme-linked immunosorbent assays (ELISAs) according to the manufacturer's instructions (catalog nos. H002, H007, H052, Jiancheng Bioengineering Institute, Nanjing, China). Concentrations were represented as $\mathrm{pg} / \mathrm{mL}$.

\section{Immunofluorescence}

Brain cryosections $(10 \mu \mathrm{m})$ from $4-5$ animals in each group were blocked with $3 \%$ bovine serum albumin and then incubated with 1:100 dilutions of primary antibodies against the following proteins: GFAP (168251-AP, Proteintech, USA), Iba-1 (ab5076, Abcam), MPO (ab9535) and ZO-1 (21773-1-AP, Proteintech). Nuclei were stained with DAPI. Immunofluorescence was observed using a BX51 fluorescence microscope (Olympus, Tokyo, Japan).

\section{Statistical analysis}

Data were expressed as mean \pm standard deviation (SD). Statistical differences were evaluated using ANOVA with post hoc Bonferroni test for groups comparison. The researchers performing statistical analysis were blinded to the treatment conditions for each dataset. All measurements were made in triplicate unless otherwise mentioned, and all experiments were performed twice. ${ }^{*} \mathrm{P}<0.05,{ }^{* *} \mathrm{P}<0.01$ and ${ }^{* * *} \mathrm{P}<0.001$.

\section{Results \\ Particle size, zeta potential, morphology, and encapsulation efficiency of PM}

The particle sizes of the PM and DPM were determined via DLS. The average diameter of PM was $123.3 \pm 18.0 \mathrm{~nm}$ at room temperature (Fig. 1a), which increased to $186.6 \pm 16.5 \mathrm{~nm}$ after encapsulation of DRC (Fig. 1b). DRC loading also changed the zeta potential of PM from $-19.33 \pm 1.59$ to $-15.21 \pm 3.68 \mathrm{mV}$ (Table 1 ). The polydispersity index (PDI) was $0.193 \pm 0.028$ for PM and $0.232 \pm 0.086$ for DPM, both of which are below 0.3 and therefore indicate narrow particle size distribution.

Transmission electron microscopy images showed that PM were uniformly spherical in shape and homogeneous in size (Fig. 1c), while size distribution of DPM micelles became larger after loaded with DRC (Fig. 1d), in line with the DLS data. Encapsulation efficiency (EE) and drug loading (DL) were calculated, respectively, as $97.10 \pm 5.21 \%$ and $10.10 \pm 1.31 \%$ (Additional file 1: Figure S3). 
Table 1 Characterizations of blank micelles (PM) and dauricine-loaded micelles (DPM)

\begin{tabular}{llllll}
\hline Sample & Diameter $(\mathbf{n m})^{\mathbf{a}}$ & Polydispersity index ${ }^{\mathbf{a}}$ & ${\text { Zeta potential }(\mathbf{m V})^{\mathbf{a}}}$ & $\begin{array}{l}\text { Encapsulation } \\
\text { efficiency (\%) }\end{array}$ & Drug loading (\%) $^{\mathbf{b}}$ \\
\hline PM & $123.3 \pm 18.0$ & $0.193 \pm 0.028$ & $-19.33 \pm 1.59$ & NA & NA \\
DPM & $186.6 \pm 16.5$ & $0.202 \pm 0.086$ & $-15.21 \pm 3.68$ & $97.10 \pm 5.21$ & $10.10 \pm 1.31$ \\
\hline
\end{tabular}

NA not applicable

$\mathrm{n}=3$, mean $\pm \mathrm{SD}$

a Based on dynamic light scattering

b Based on HPLC

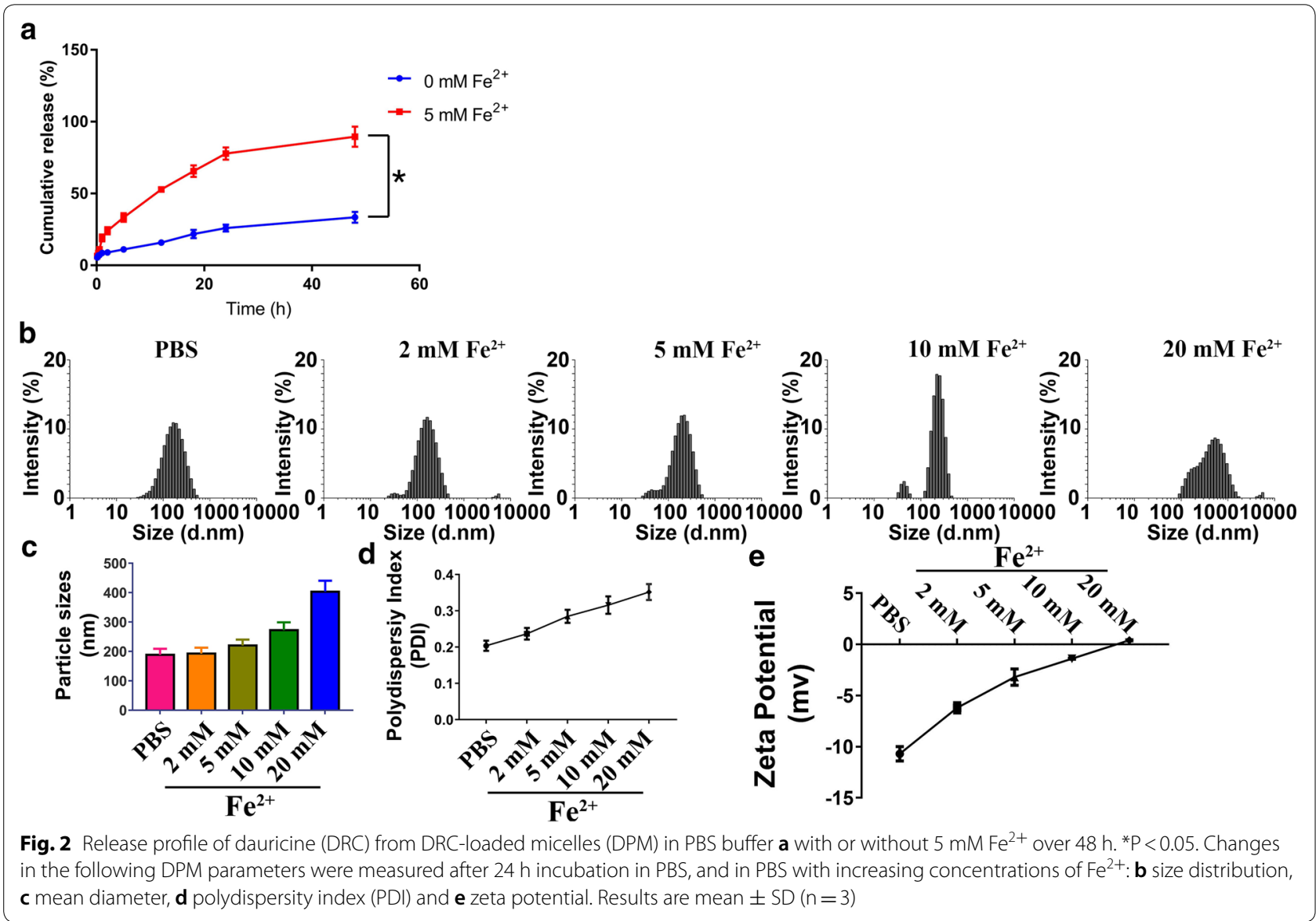

\section{In vitro release of DRC from DPM}

The DRC release profiles from DPM in PBS ( $\mathrm{pH} 7.4$ ) with or without $5 \mathrm{mM} \mathrm{Fe}{ }^{2+}$ are shown in Fig. 2a. DPM was stable in PBS without $\mathrm{Fe}^{2+}$, showing only about $25 \%$ release of DRC after $24 \mathrm{~h}$ at $37{ }^{\circ} \mathrm{C}$. In the presence of $5 \mathrm{mM} \mathrm{Fe}^{2+}$, in contrast, more than $24 \%$ of the DRC load was released from DPM within $2 \mathrm{~h}$ and about $80 \%$ by $24 \mathrm{~h}$. DPM maintained a stable mean diameter of $189.8 \pm 20.5 \mathrm{~nm}$ and narrow size distribution during 24-h incubation in PBS (Fig. 2b), which did not change substantially for at least $96 \mathrm{~h}$ (Additional file 1: Figure
S4). These results indicate that DPM can remain intact for long periods in the absence of $\mathrm{Fe}^{2+}$, and then release DRC selectively in response to high $\mathrm{Fe}^{2+}$ concentrations. It has been proposed that $\mathrm{Fe}^{2+}$ can neutralize the anionic head groups of phosphonates in PM, destabilizing the micelles and accelerating DRC release. Consistent with this idea, we found that adding $\mathrm{Fe}^{2+}$ to PM or DPM increased mean micelle size, polydispersity index and zeta potential (Fig. 2c-e). 


\section{Neuroprotective effects of DPM}

The therapeutic potential of DPM was analyzed in vitro, based on studies implicating massive release of metal ions such as $\mathrm{Fe}^{2+}$ and high levels of neurotoxic ROS in the neuronal cell injury and apoptosis of $\mathrm{ICH}$ [27]. Figure 3 and Additional file 1: Figure S5 show the protective potential of different micelle formulations against $\mathrm{Fe}^{2+}$ and $\mathrm{Cu}^{2+}$-mediated effects on SH-SY5Y cells. Addition of $5 \mathrm{mM} \mathrm{Fe}^{2+}$ reduced SH-SY5Y cell viability by about $50 \%$ (Additional file 1: Figure S6), while free DRC could only slightly reverse this, DPM significantly increased the cell viability (Fig. 3), with assistance of good biocompatible PM (Additional file 1: Figure S7). Similar results were observed with the addition of $\mathrm{Cu}^{2+}$ (Additional file 1: Figure S5).

\section{Ability of DPM to inhibit ROS production}

We compared the ability of free DRC, PM and DPM to reduce $\mathrm{Fe}^{2+}$-induced intracellular ROS generation, measured as DCF fluorescence (Fig. 4). ROS production was lower with any of the three treatments than with vehicle, and it was lowest with DPM (7.2-fold lower than vehicle). This large inhibition of ROS production may reflect the ability of DPM not only to protect neurons but also to chelate the toxic $\mathrm{Fe}^{2+}$.

\section{Ability of DPM to inhibit apoptosis}

Apoptosis was induced in SH-SY5Y cells by $5 \mathrm{mM} \mathrm{Fe}^{2+}$, which led to $17.37 \pm 2.73 \%$ of apoptotic cells. Treatment with $1 \mu \mathrm{M}$ DPM reduced this fraction to $3.11 \pm 0.37 \%$, lower than the fraction of $5.43 \pm 0.64 \%$ with free DRC and much lower than the fraction of $10.19 \pm 1.06 \%$ with PM (Fig. 5).

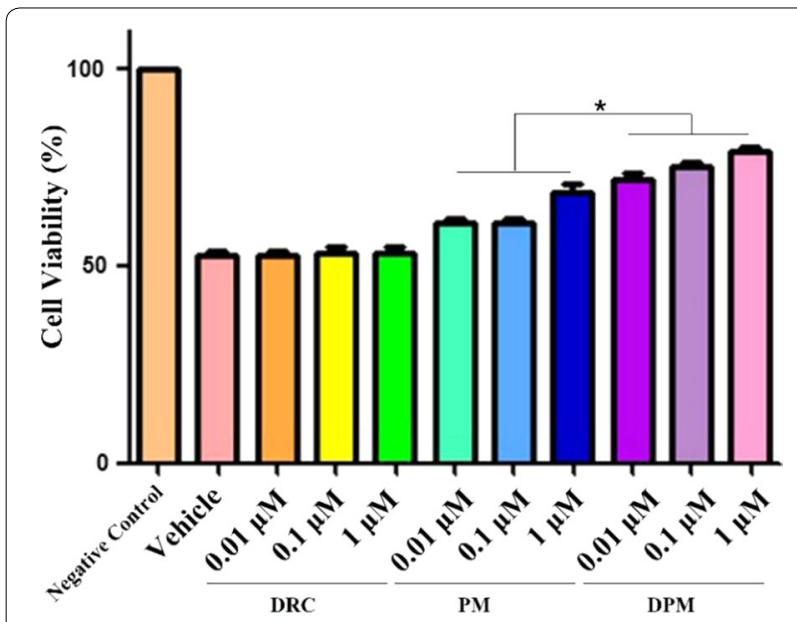

Fig. 3 Neuroprotective effects of DPM against toxicity of $5 \mathrm{mM} \mathrm{Fe} \mathrm{F}^{2+}$ in SH-SY5Y cells. Cells were treated with free DRC, blank micelles (PM) or dauricine-loaded micelles (DPM). ${ }^{*} \mathrm{P}<0.05(n=5)$

\section{Ability of DPM to improve ICH-induced neurological} deficits

Before $\mathrm{ICH}$, mNSS was 0 in all animals, and the inducement of $\mathrm{ICH}$ significantly increased (worsened) this score. Treatment for $24 \mathrm{~h}$ with DPM led to a significantly lower score than treatment with DRC $(7.63 \pm 1.30$ vs $9.50 \pm 1.41, \mathrm{p}<0.05$; Fig. 6). In addition, DPM animals scored better than any other group of $\mathrm{ICH}$ animals in paw placement test and corner turn test (Additional file 1: Figure S8).

\section{Ability of DPM to alleviate brain edema and loss of blood- brain barrier permeability after ICH}

To evaluate effects of DPM on brain edema after $\mathrm{ICH}$, brain water content was examined in contralateral and ipsilateral cortices following treatment at $24 \mathrm{~h}$ after $\mathrm{ICH}$ (Fig. 7a). DPM was associated with significantly lower brain water content than free DRC in the contralateral cortex $(73.80 \pm 1.89 \%$ vs $75.55 \pm 1.59 \%, \mathrm{P}<0.05)$ and ipsilateral cortex $(74.36 \pm 1.95 \%$ vs $75.45 \pm 1.61 \%, \mathrm{P}<0.05)$.

To investigate the effects of DPM on $\mathrm{ICH}$-induced permeabilization of the blood-brain barrier, an Evans blue extravasation assay was performed at $24 \mathrm{~h}$ after $\mathrm{ICH}$. DPM was associated with significantly less Evans blue leakage than free DRC $(26.05 \pm 1.93$ vs $32.77 \pm 2.96 \mu \mathrm{g} / \mathrm{g}$, $\mathrm{P}<0.05$, Fig. 7b).

Taken together, these results suggest that DPM mitigates $\mathrm{ICH}$-induced neurological deficits, brain edema, and blood-brain barrier permeabilization.

\section{Accumulation of micelles at hematoma sites}

We hypothesized that the micelles would preferentially release their cargo at sites of hematoma injury because the negative charges on the micelle surface should be neutralized by abundant metal ions in the surroundings, as confirmed in vitro (Fig. 9d). To test this, we examined the distribution of micelles in ex vivo organs from treated animals at 12 and $24 \mathrm{~h}$ post-injection. To enable micelle tracking, we loaded PM with the near-infrared fluorescent dye DiR and injected the micelles into mice via the tail vein immediately after ICH. DiR signals in organs of both cases at different time points were shown in Fig. 8a. The proportion of DiR signal in the brain was much higher in DPM-treated mice than in animals treated with free dye after $24 \mathrm{~h}$ (Fig. 8b). These results suggest that, as desired, DPM tends to accumulate, and release its DRC cargo, at sites of ICH injury in the brain.

\section{Ability of DPM to alleviate ICH-induced brain damage in a mouse model of autologous blood ICH}

Mice were subjected to MRI examination following treatment at $24 \mathrm{~h}$ after $\mathrm{ICH}$ induction. T2-weighted MRI 

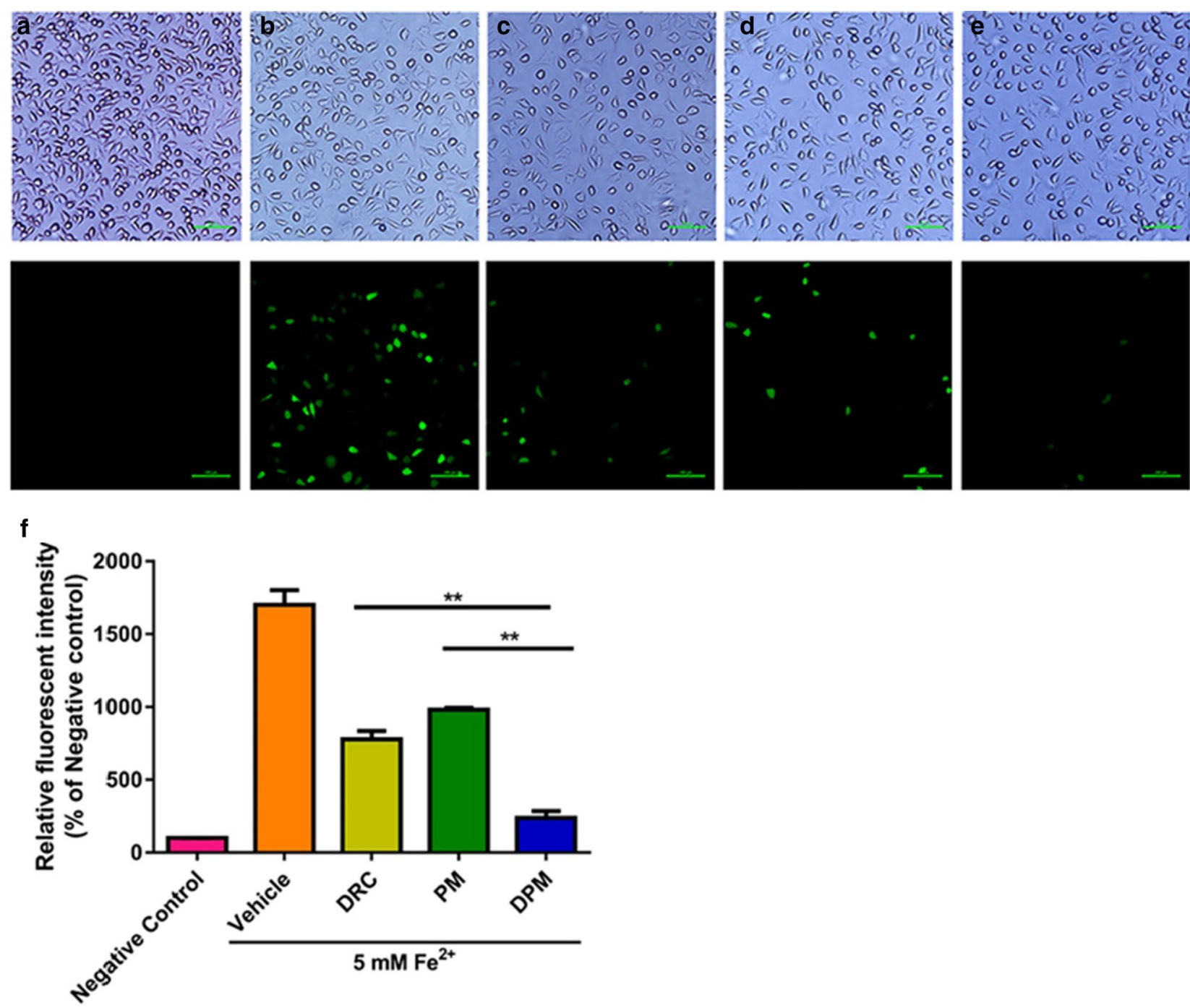

Fig. 4 Representative micrographs of SH-SY5Y cultures treated with a nothing (cell culture medium only), b vehicle (DMSO), c DRC (1 $\mu M)$, d PM $(5 \mu M)$, or e DPM $(1 \mu M)$. f Histogram of intracellular ROS levels after exposure to the various formulations. Scale bar $100 \mu m{ }^{* *} P<0.01(n=3)$

showed obvious brain hematoma, edema and injury in all mice. The MRI signal was mixed and included hypointense signal, suggestive of hematoma tissue, as well as a hyperintense signal surrounded by dark rims, suggestive of perihematoma edema. DPM mitigated these effects of ICH to a greater extent than free DRC (Fig. 9a). These effects of ICH and the neuroprotective effects of DPM were confirmed by staining brain tissues with hematoxylin-eosin and cresyl violet (Fig. 9b, c).

The effects of DPM were associated with a reduction in ferric iron deposition (as blue color indicating), based on Perl's staining of brain sections (Fig. 9d). Iron deposition is triggered by $\mathrm{ICH}$ and induces production of ROS and lipid hydroperoxides to lethal levels, known as ferroptosis [28].

\section{Ability of DPM to reverse ZO-1 down-regulation}

We used immunofluorescence staining and western blotting to detect the expression of $\mathrm{ZO}-1$ at the tight junctions in the brains of animals at $24 \mathrm{~h}$ after $\mathrm{ICH}$ induction. ICH down-regulated the ZO-1 protein, and DPM reversed this to a significantly greater extent than PM or free DRC (Fig. 10a). Similar results were observed in western blots (Fig. 10b, c).

\section{Ability of DPM to inhibit ICH-induced apoptosis and ferroptosis}

The balance between Bcl-2 and Bax levels determines the balance between cell survival and death. While higher expression of $\mathrm{Bcl}-2$ inhibits apoptosis, higher expression of Bax induces mitochondrial membrane 


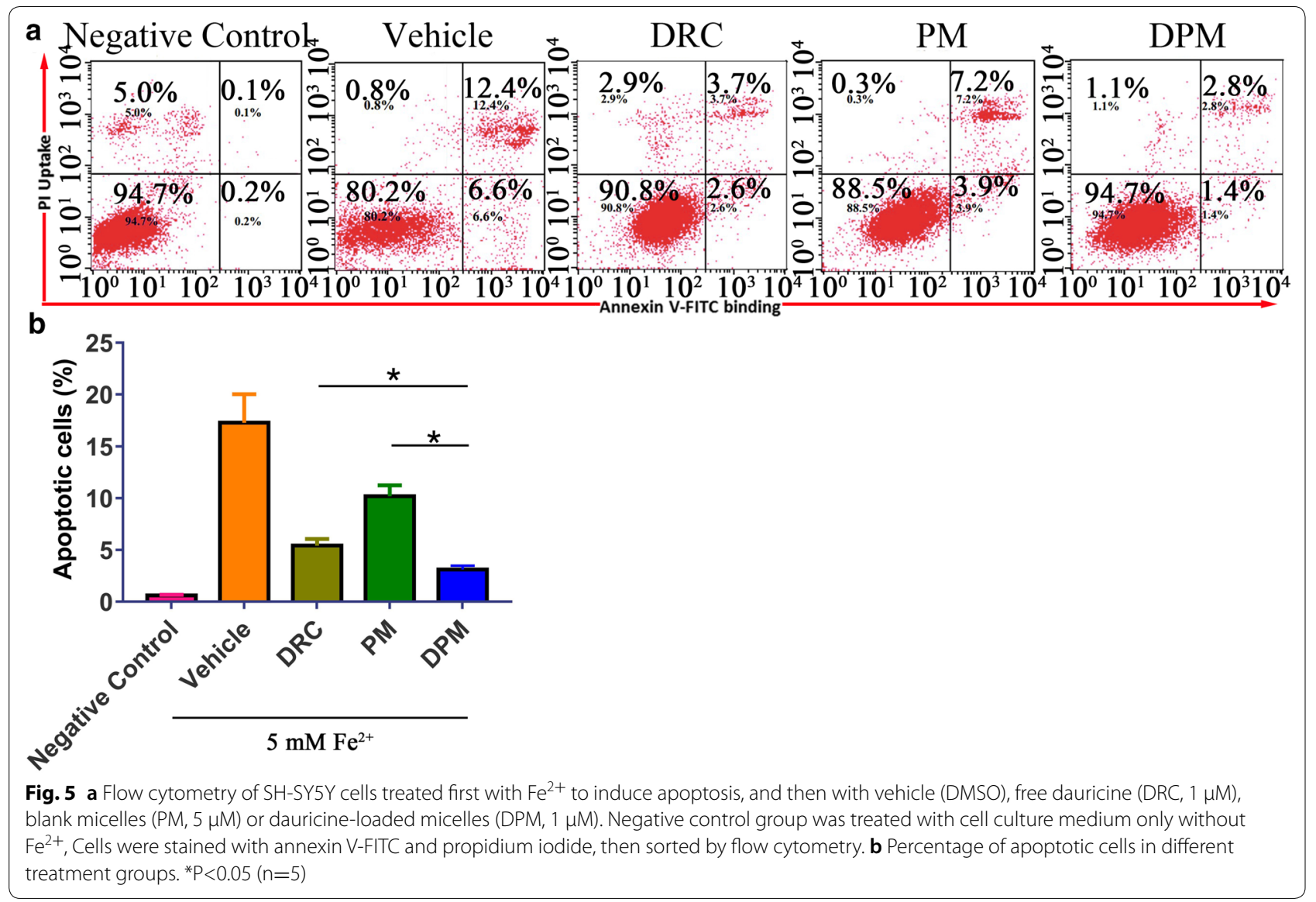

permeabilization, release of cytochrome c, and activation of caspases-9 and -3, initiating apoptosis. Compared to sham surgery, ICH significantly decreased Bcl-2 expression and increased Bax and caspase- 3 expression in the left hemisphere (Fig. 11a). DPM (100 mg/kg) reversed

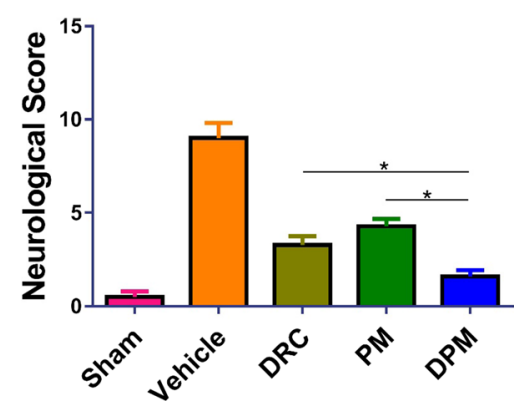

Fig. 6 Assessment of neurological defects in mice at $24 \mathrm{~h}$ after $\mathrm{ICH}$ and treatment with vehicle (0.9\% saline), free dauricine (DRC), blank micelles (PM) or dauricine-loaded micelles (DPM). The sham group was set up by performing craniotomy without blood infusion. Animals were scored using the modified neurological severity score. Results are mean $\pm S D$ ( $n=10$ per group). ${ }^{*} \mathrm{P}<0.05$ these effects, increasing the Bcl-2/Bax ratio (Fig. 11b, c). At the same time, DPM up-regulated glutathione peroxidase 4 (GPX4), and this enzyme down-regulates ferroptotic cell death by converting toxic lipid hydroperoxides into non-toxic lipid alcohols (Fig. 11b). PM and free DRC also up-regulated GPX4, although to a smaller extent.

\section{Ability of DPM to attenuate microglial activation, astrocyte activation, and neutrophil infiltration after $\mathrm{ICH}$}

To further examine additional mechanisms by which DPM might attenuate ICH injury, we assessed microglial activation, astrocytic activation, and neutrophil infiltration in perihematomal brain tissue at $24 \mathrm{~h}$ after $\mathrm{ICH}$. Microglia were stained with Iba-1 immunofluorescence and their morphology examined: resting or quiescent microglia appear long, thin, and rod-like, while activated microglia have enlarged cell bodies with short processes. DPM led to significantly fewer activated microglia in perihematomal tissue (Fig. 12a). Astrocytic activation was assessed by observing GFAP-positive cells. DPM led to significantly less astrocytic activation in perihematomal tissue than free DRC or PM (Fig. 12b). Based on MPO staining, we found significantly fewer infiltrating 


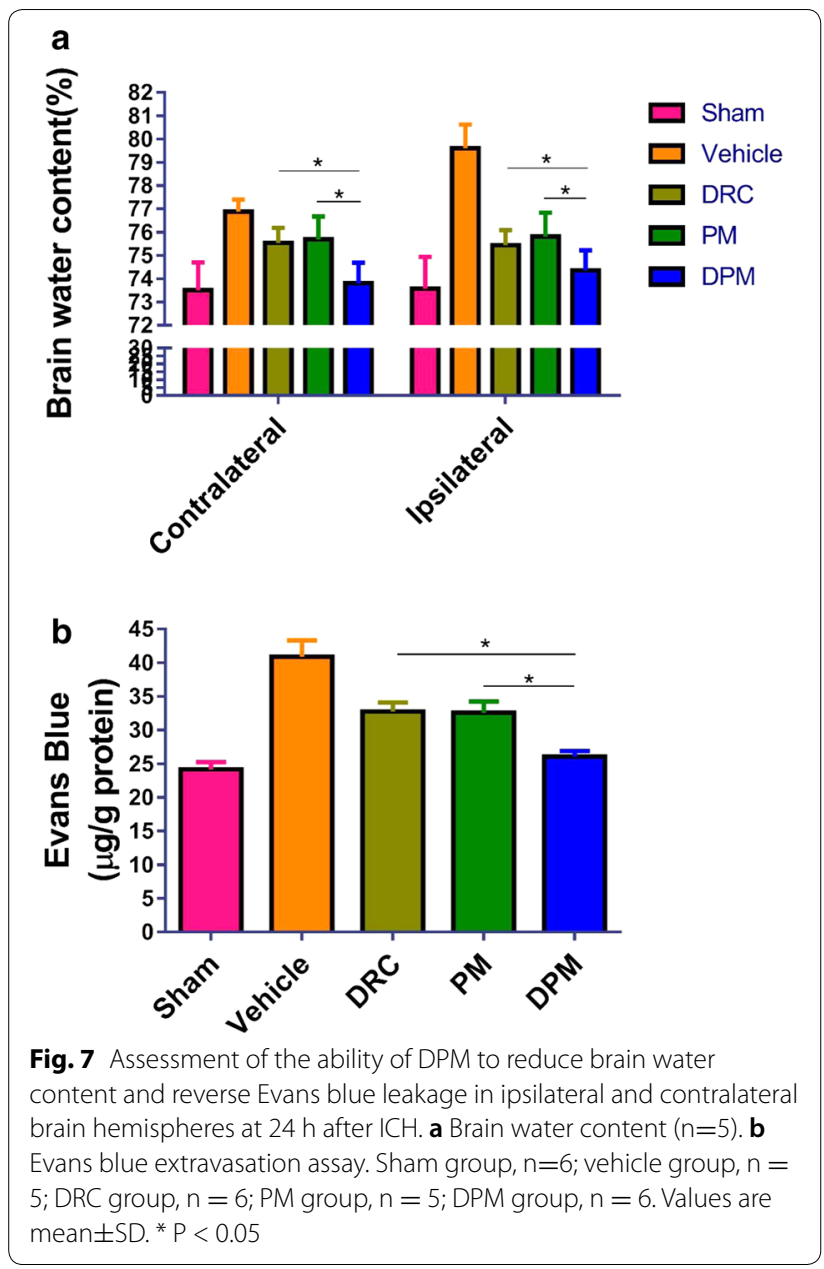

neutrophils in the perihematoma of the DPM group than in the other groups (Fig. 12c).

\section{Ability of DPM to reduce ICH-induced production of IL-1 $\beta$, IL-6, TNF- $\alpha$ and MMP-9}

To investigate the anti-inflammatory effects of DPM in $\mathrm{ICH}$, the levels of pro-inflammatory factors IL-1 $\beta$, IL-6, and TNF- $\alpha$ as well as MMP-9 in the perihematomal brain tissue were measured. As expected, ICH up-regulated the levels of all four factors, and DPM significantly reversed these effects (Fig. 13).

\section{Discussion}

Intracerebral hemorrhage $(\mathrm{ICH})$ occurs when a weakened vessel ruptures and bleeds into the surrounding brain [29, 30]. Various forms of cell death have been identified after $\mathrm{ICH}$, including apoptosis, necrosis, and autophagy in humans and experimental animals, and autophagic cell death in animal models [31-33]. Although inhibiting apoptosis, necrosis, and

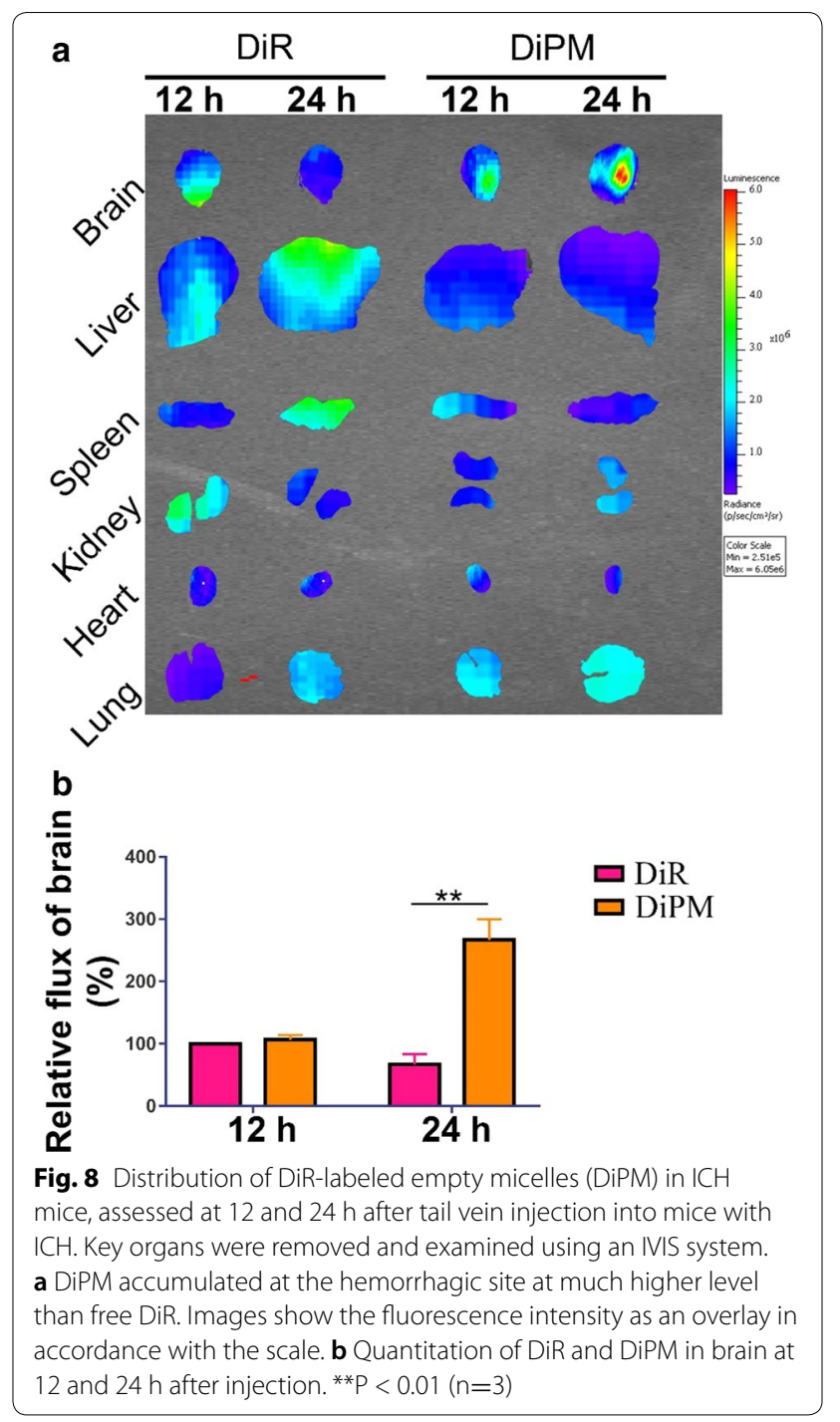

autophagy can improve outcomes in animals subjected to experimental $\mathrm{ICH}$, no successful clinical trials using cell-death inhibitor monotherapy have been reported [34-36]. These facts suggest that multiple forms of cell death other than those mentioned above may occur after $\mathrm{ICH}$ and contribute collectively to neuronal death.

Toxins released from an intracerebral hematoma may contribute to brain damage after $\mathrm{ICH}$ [37]. Two putative neurotoxins are hemoglobin, the most abundant protein in blood, and its heme group, which are released from lysed erythrocytes after ICH [38-40] Hemoglobin and its heme group play an essential role in ROS production after ICH [41-43]. In addition, hemoglobin can be metabolized into ferrous/ferric iron and form hydroxyl radicals via the Fenton reaction [44]. These highly toxic radicals attack DNA, proteins, and lipid membranes, leading to ferroptosis and production 


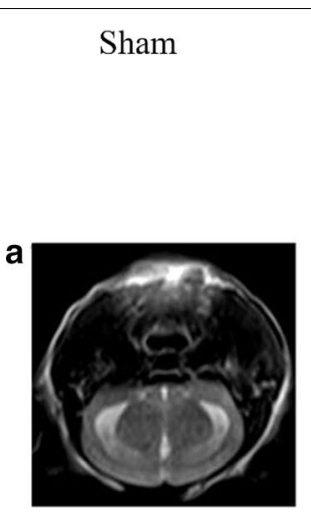

b

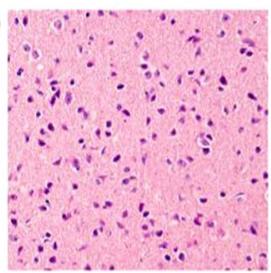

c.

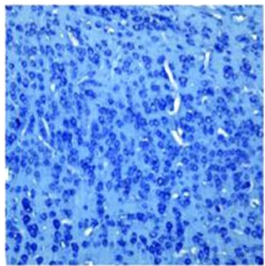

d

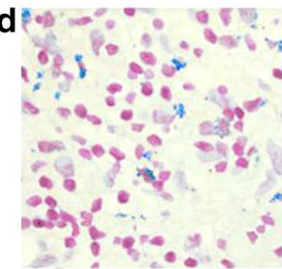

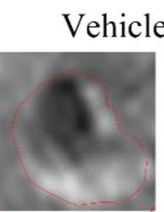
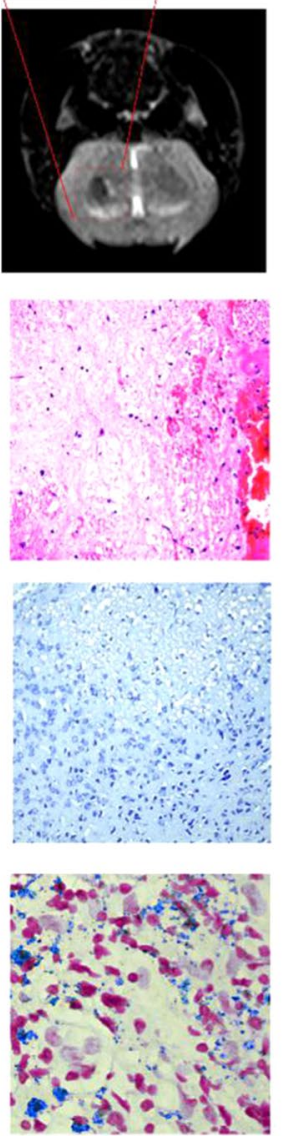

DRC
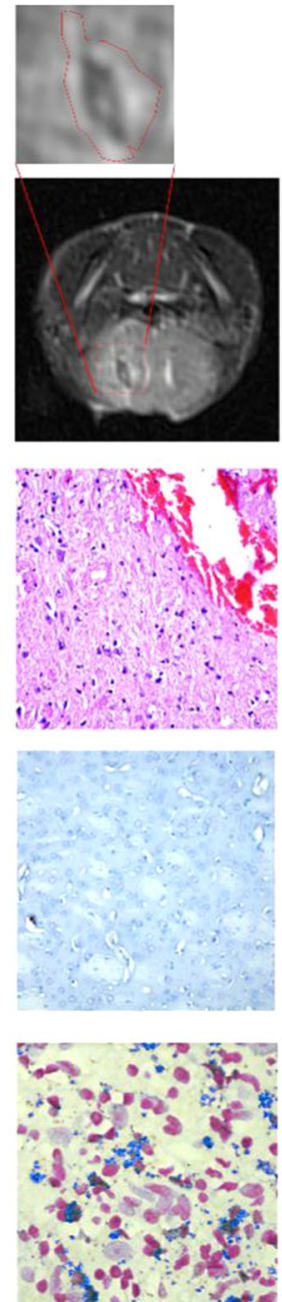

$\mathrm{PM}$
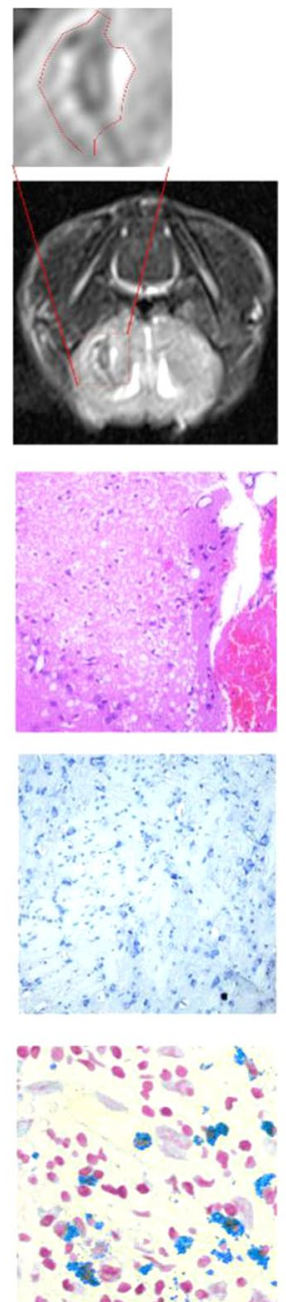

DPM
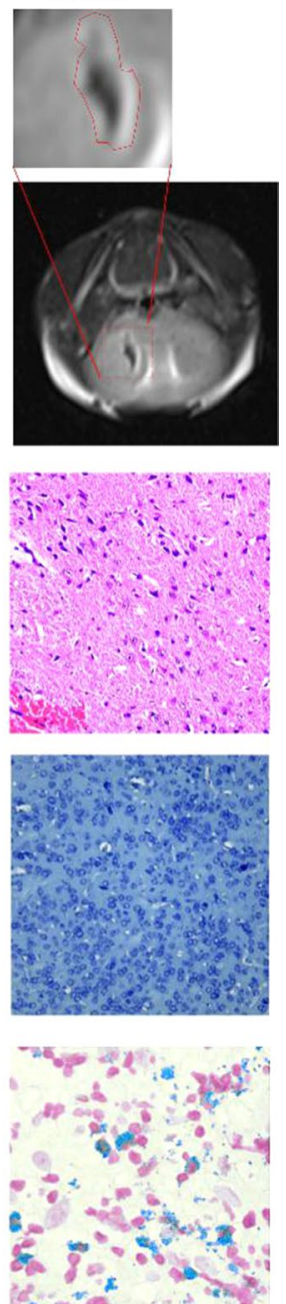

Fig. 9 Effect of DPM on ICH-induced brain damage in a mouse model of autologous blood ICH. a T2-weighted turbo spin echo magnetic resonance images after $24 \mathrm{~h}$ of false ICH operation (sham), 0.9\% saline (vehicle), free dauricine (DRC), empty micelles (PM) or dauricine-loaded micelles (DPM). The areas demarcated with dash lines contain a bright rim surrounding the hematoma due to edema formation and/or extruded serum from the injected blood. $\mathbf{b}$ Representative sections of brain tissue stained with hematoxylin-eosin. Magnification, $\times 100$. $\mathbf{c}$ Representative sections of brain tissue stained with cresyl violet. Magnification, $\times 20$. $\mathbf{d}$ Representative sections of brain tissue stained with Perl's stain to detect iron. Magnification, $\times 200$

of pro-inflammatory factors $[45,46]$. This recently described form of cell death plays a major role in tumor development and embryonic development and also contributes to neuronal death in a mouse model of $\mathrm{ICH}$ $[47,48]$.

These considerations suggest that effective $\mathrm{ICH}$ treatments should target the multiple injury pathways involved, including apoptosis, iron deposition and ferroptosis, ROS as well as inflammation $[49,50]$. The present study shows that micelles assembled from a calix[4] arene scaffold with hydrophobic $n$-dodecyl chains can act as an $\mathrm{Fe}^{2+}$-responsive delivery vehicle for DRC to inhibit all of these ICH-induced damage pathways selectively at sites of brain injury. In our experiments, DPM decreased ROS levels in vitro, increased the Bcl-2/Bax ratio in brain tissues, and inhibited ferroptosis. These effects were associated with improvement in ICH-induced neurological defects. Our results suggest that DPM can alleviate primary and secondary brain injury driven by apoptosis and ferroptosis.

Among the macromolecules, calixarene is considered to represent the third-generation of host-guest supramolecular chemistry [51]. Calixarene and its derivatives have been reported to have antiviral, antibacterial, antifungal, 


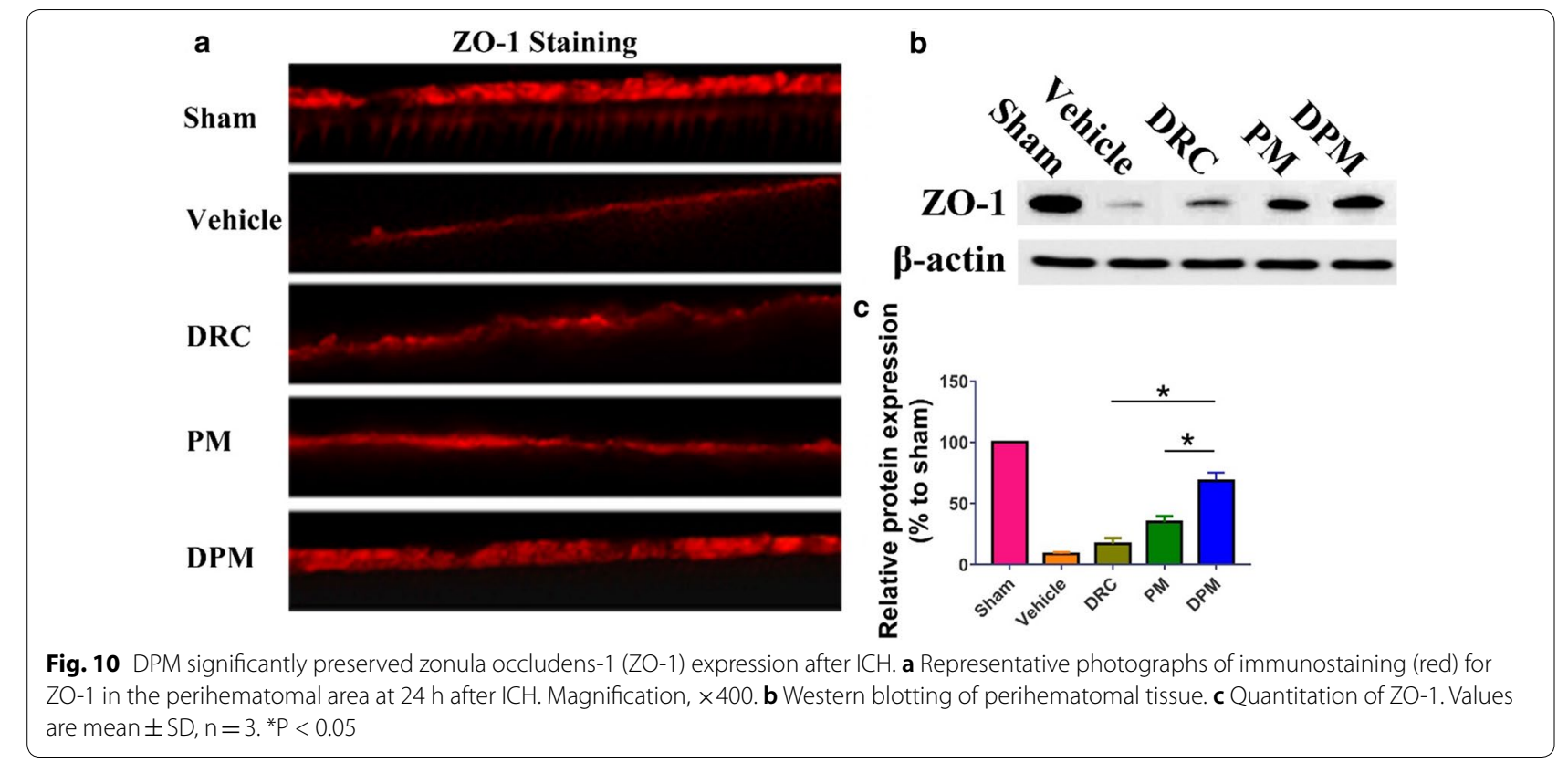

b
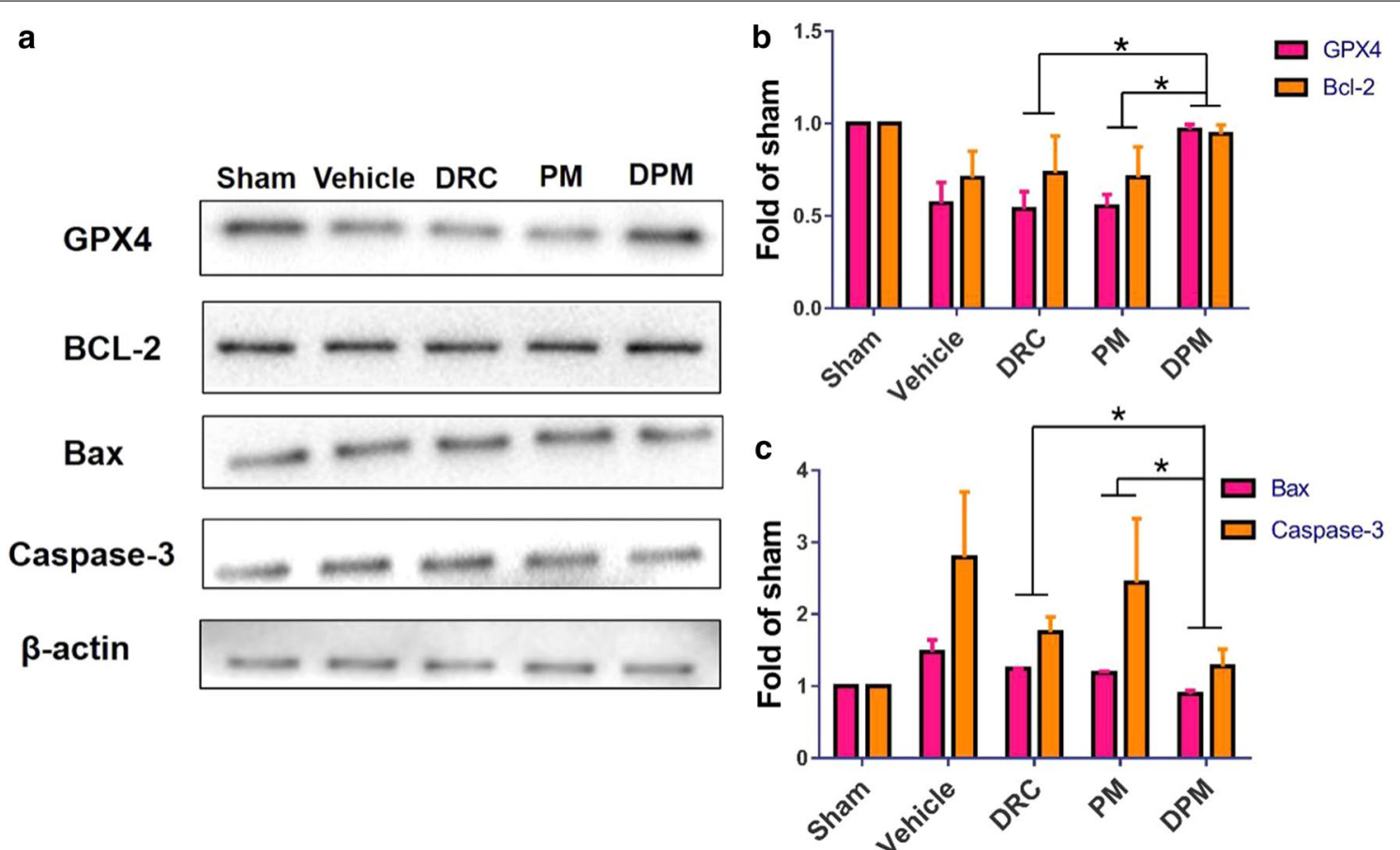

Fig. 11 Effects of DPM on ICH-induced apoptosis and ferroptosis. a Representative Western blots of GPX-4, BCl-2, Bax, and caspase-3 in the left hemisphere of at $24 \mathrm{~h}$ after $\mathrm{ICH}$ and treatment with nothing (sham), $0.9 \%$ saline (vehicle), free dauricine (DRC), empty micelles (PM) or dauricine-loaded micelles (DPM). Results are representative of three experiments. Levels of $\mathbf{b}$ GPX 4 and BCl-2 as well as $\mathbf{c}$ Bax and caspase-3 were quantitated relative to $\beta$-actin, and levels are expressed relative to that in the sham group. Values are mean $\pm S D, n=3 .{ }^{*} P<0.05$

antitubercular, and anticancer activity [52]. In the present work, from TEM images, we found that the size of PM and ad DPM are same as DLS. As we know Dynamic light scattering (DLS) was used to determine the hydrodynamic diameters of nanoparticles. Therefore, for inorganic nanoparticles, their DLS sizes are much higher 
a
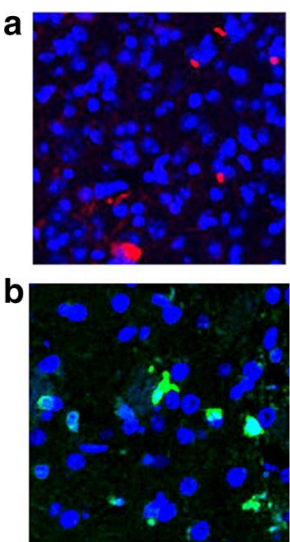

C

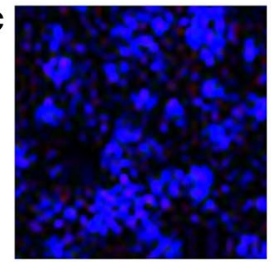

d

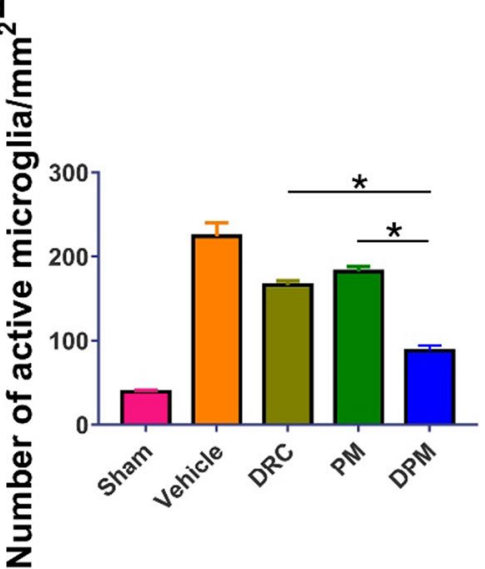

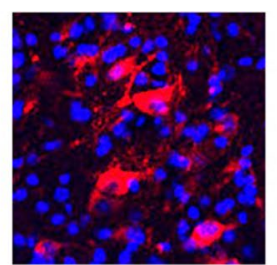
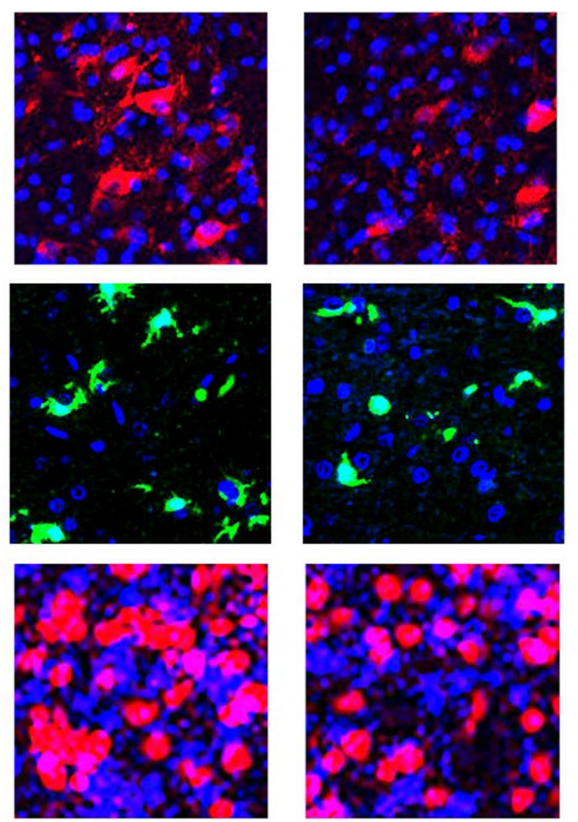
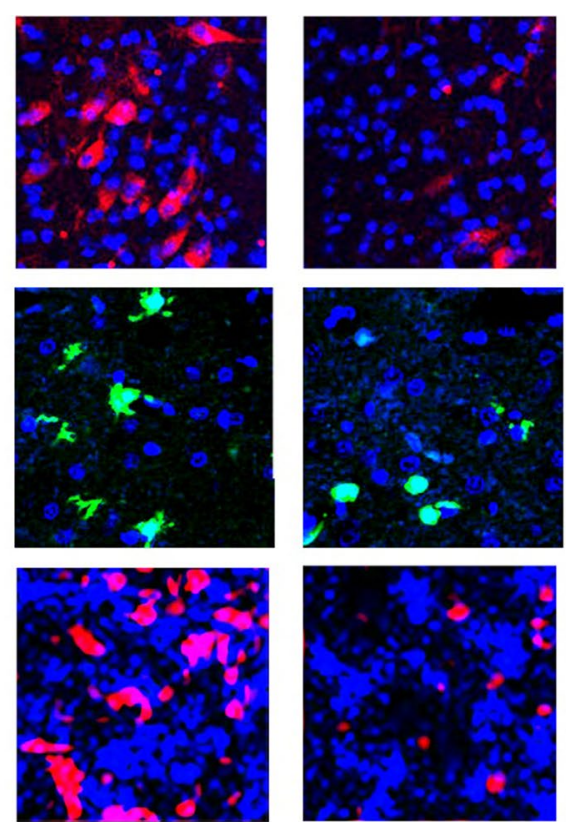

\begin{abstract}
e
\end{abstract}

\title{
f
}
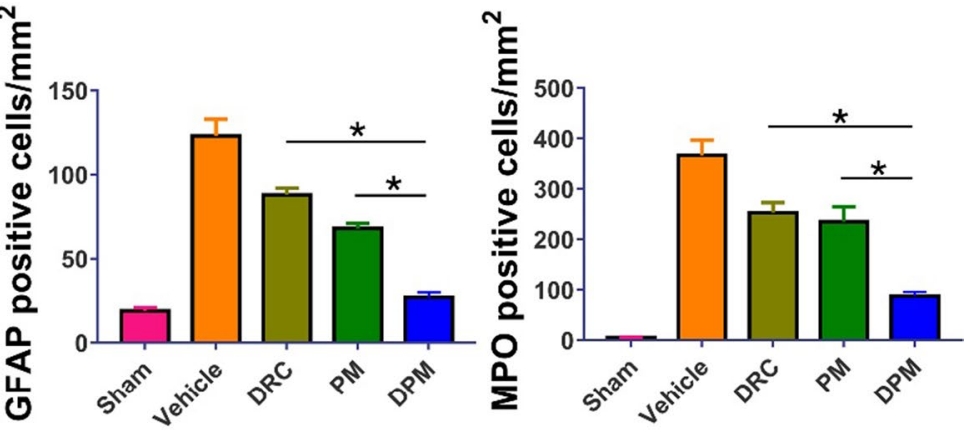

Fig. 12 Ability of DPM to partially reverse ICH-induced activation of microglia and astrocyte as well as infiltration by neutrophils. Brain sections were prepared at $24 \mathrm{~h}$ after $\mathrm{ICH}$ and immunostained to detect the three cell populations. a Representative photomicrographs of brain tissue immunostained against Iba-1 to reveal microglia. Magnification, $\times 100$. b Representative photomicrographs of brain tissue immunostained against GFAP to reveal astrocytes. Magnification, $\times 200$. c Representative photomicrographs immunostained against MPO to reveal neutrophils. Magnification, $\times 100 . \mathbf{d}-\mathbf{f}$ Quantitation of the experiments shown in panels $(\mathbf{a}-\mathbf{c})$, respectively. Values are $m e a n \pm S D, n=3 .{ }^{*} P<0.05$

than their TEM sizes, as the hydration shell around inorganic nanoparticles would evaporate after samples on TEM copper grid were dried under room temperature. However, cases would be different with organic nanoparticles, whose shape could change from $3 \mathrm{D}$ sphere to $2 \mathrm{D}$ thin film upon losing water outside and inside nanoparticles after drying under room temperature. In our previous research, we confirmed by atomic force microscopy (AFM) that nanoparticles would partially collapse, leading to about $20 \%$ larger size than that of DLS size [18]. Herein, for TEM images of PM and DPM, we care more about their morphology and sizes distribution. Similar discrepancy was observed by Yao Wang et al. who found that the size of Mac-1 with DOX from TEM images was around $30 \mathrm{~nm}$ in average, while most of the nanogel particles remained at smaller radius with the peak around $10 \mathrm{~nm}$ from DLS analysis [53].

Moreover, Calix[4]arene, bearing methylenebisphosphonic groups, can host cationic small drugs such as carboplatin or metal ions such as $\mathrm{Cu}^{2+}$ or $\mathrm{Fe}^{2+}$ in its cavity $[10,16,17]$. In previous work, we showed that micelles similar to PM in the present study delivered anti-cancer drugs in response to $\mathrm{pH}[54,55]$. In that study, the micelles had shorter $n$-hexyl chains, which we lengthened 
a

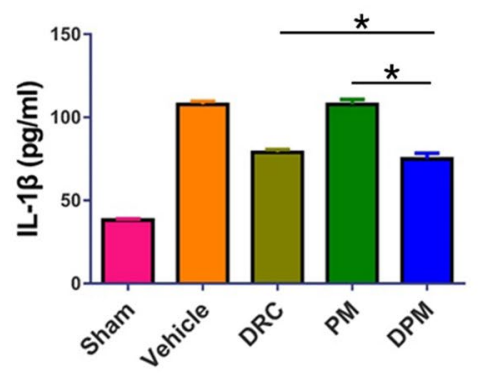

d

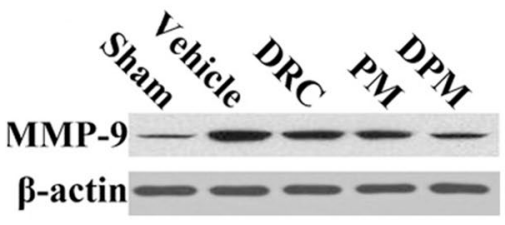

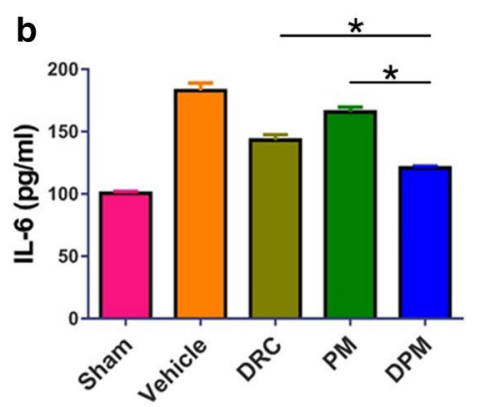

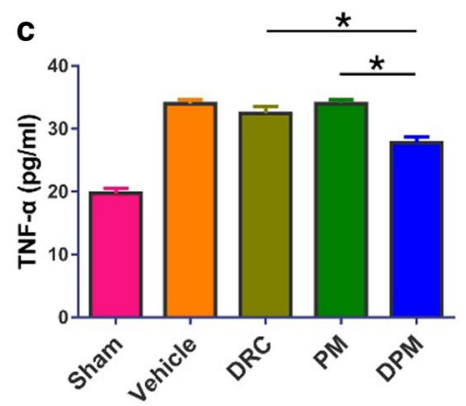

e

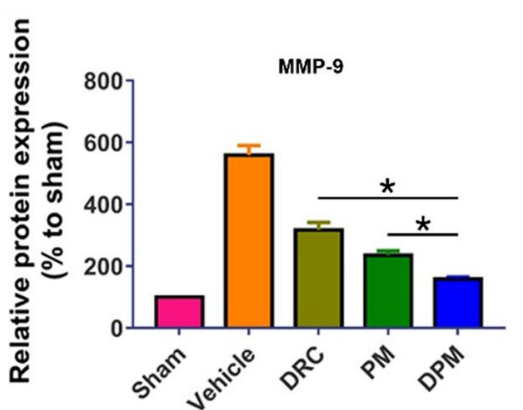

Fig. 13 Effect of DPM on the ICH-induced pro-inflammatory state in the perihematomal area. Animals were subjected to ICH and treatment with 0.9\% saline (vehicle), free dauricine (DRC), empty micelles (PM) or dauricine-loaded micelles (DPM). The sham group was set up by performing craniotomy without blood infusion. At $24 \mathrm{~h}$ later, brain tissue was isolated and assayed by ELISA for a IL-6, b IL-1 $\beta$ and $\mathbf{c}$ TNF-a, or by Western blot for d, e MMP-9. Values are mean $\pm \mathrm{SD}(\mathrm{n}=3) .{ }^{*} \mathrm{P}<0.05$

to $n$-dodecyl chains for this study to create larger micelles for higher drug loading. The present work further demonstrates the flexibility of the calix[4]arene scaffold for delivering drugs in response to specific stimuli. The micelles in the present study respond to high concentrations of metal cations. Since the pKa of the phosphonic acid head groups is 7.21, the head groups should be deprotonated at physiological $\mathrm{pH}$ of 7.4, and this charge may be neutralized by metal ions such as $\mathrm{Fe}^{2+}$ and $\mathrm{Cu}^{2+}$. Such neutralization may destabilize the micelles, accelerating release of the drug cargo. This may explain how the micelles can release drug preferentially in perihematomal tissue, where metal ion concentrations are elevated [56, 57].

And in our experiments, we chose $5 \mu \mathrm{M}$ PM for further experiment.As shown in Fig. 3, optimized dose of DRC, PM and DPM was investigated by incubation of a serial of concentrations of DRC, PM and DPM with SHSY5Y cells, with all treatment groups also receiving $\mathrm{Fe}^{2+}$ to a final concentration of $5 \mathrm{mM} .1 \mu \mathrm{M}$ of DRC and DPM were chosen for further experiments since the discrepancy of cell viabilities is the hugest on this concentration. As calculated above, $1 \mu \mathrm{mol}$ of DPM contains $5562.2 \mu \mathrm{g}$ of $p$-PCa4C12, which equal to $3.8 \mu \mathrm{mol} p$-PCa4C12. To increase the redundancy, higher concentration $(5 \mu \mathrm{M})$ of $p$-PCa4C12 micelles (PM) was used to compare with DPM for their treatment efficacies in ICH. Thus, $5 \mu \mathrm{M}$ PM was correspondingly chosen for further experiment.

The reason why the concentrationof $\mathrm{Fe}^{2+}$ was determined in vitro is that in our preliminary experiment, toxicities of different concentrations of $\mathrm{Fe}^{2+}$ to SH-SY5Y cells after co-incubation for $24 \mathrm{~h}$ was evaluated, we found $5 \mathrm{mM} \mathrm{Fe}{ }^{2+}$ could inflict $40 \%$ of cells death (Additional file 1: Figure S6). Higher concentration of $\mathrm{Fe}^{2+}$ as $10 \mathrm{mM}$ would cause $55 \%$ cell deceased, which is too severe to be recovered by DPM. And lower concentration of $\mathrm{Fe}^{2+}$ as $1 \mathrm{mM}$ would lead to $24 \%$ cell died, which is too mild to distinguish efficacies between DRC and DPM. Therefore, the concentration of $5 \mathrm{mM} \mathrm{Fe}^{2+}$ was deemed as appropriate concentration for further experiments. Cytotoxicity of $\mathrm{Fe}^{2+}$ varied significantly in different research groups. Some researcher group got similar result as what we got in terms of $\mathrm{IC}_{50}$ of $\mathrm{Fe}^{2+}$. Takahiko Imai et al. revealed $300 \mu \mathrm{M} \mathrm{Fe}{ }^{2+}$ caused about $4 \%$ endothelial cells death after a $24 \mathrm{~h}$ incubation period, which translated to $\mathrm{IC}_{50}$ of $3.8 \mathrm{mM}$ for $\mathrm{Fe}^{2+}$ [58]. Considering different lab's conditions and cell lines, $5 \mathrm{mM} \mathrm{Fe}{ }^{2+}$ inflicting $40 \%$ of cells death for in vitro experiments is plausible.

The FACS results in Fig. 5 show that there is only $15-17 \%$ apoptotic cells in the vehicle treated group, 
whereas the cell viability studies using CCk-8 shows much higher apoptosis, assuming at the same dose of $5 \mathrm{mM} \mathrm{Fe}^{2+}$ concentration. The main reason for the discrepancy between the results of cell viability and apoptosis is the incubation time is different. The incubation time for cell viability is $24 \mathrm{~h}$, while the incubation time is only $12 \mathrm{~h}$ for apoptosis experiment. More importantly, in apoptotic test, after cultured for $12 \mathrm{~h}$ in the presence of $5 \mathrm{mM} \mathrm{Fe}^{2+}$ (to induce apoptosis) as well as $1 \mu \mathrm{M}$ DRC, $5 \mu \mathrm{M}$ PM or DPM (final DRC concentration, $1 \mu \mathrm{M}$ ), SHSY5Y cells were incubated another $24 \mathrm{~h}$ to recover with fresh DMEM without any treatment or $\mathrm{Fe}^{2+}$.

$\mathrm{DiR}$ is a small hydrophobic molecule, which have shorter Tmax (time for peak of blood concentration) and then rather quickly excrete from kidney. In our research, DiR solution mainly accumulated in the liver, spleen and lungs after tail vein injection, which is consistent with literature [59]. Forming DiR loaded micelles usually would dramatically change its pharmacokinetic profiles, as shown in our in vivo results. After $24 \mathrm{~h}$ of tail vain injection, DiPM preferably accumulated in brain and lung, while its distribution reduced in other organs. The possible reasons for this phenomenon are as following: Firstly, DiPM would preferentially release DiR at sites of hematoma injury because the negative charges on the micelle surface would be neutralized by abundant metal ions in the surroundings. Furthermore, as shown in vitro releasing experiment (Fig. 2a), percentage of cumulative release will reach plateau after $24 \mathrm{~h}$, which may explain higher accumulation of DiPM in $24 \mathrm{~h}$ than that in $12 \mathrm{~h}$. Secondly, the accumulation of these DiPM in lung likely reflects their large size and persistence in systemic circulation. Consistent with other researcher has reported that particles $<20 \mathrm{~nm}$ are susceptible to clearance through kidneys while nanoparticles will continuous accumulated in the lung as their size increased [60]. Therefore, the intensity of fluorescence was higher in the lung in $24 \mathrm{~h}$ than that of $12 \mathrm{~h}$. Finally, the fluorescent intensities of DiPM are negligible in either liver or spleen in both $12 \mathrm{~h}$ and $24 \mathrm{~h}$, which may suggest DiPM could effectively escape the capture of RES (reticulo-endothelial system) in the liver and spleen. Thus, the concentrations of DiPM in both brain and lung were much higher than those in treatment group of DiR, considering much less accumulation of DiPM in other organs.

Blood-Brain Barrier (BBB) disruption is a hallmark of ICH-induced brain injury [61]. Therefore, after systemically administration of DPM, DPM will reach the hemorrhagic site after they pass through the ruptures in the BBB. Furthermore, after DPM chelating with metal ions including $\mathrm{Fe}(\mathrm{III})$, DPM may be further transferred across $\mathrm{BBB}$ by transferrin receptor 1 , which is highly expressed by brain capillary endothelial cells (BCECs) $[62,63]$. In the current research, DPM significantly reduced brain water content of both hemispheres at $24 \mathrm{~h}$ after $\mathrm{ICH}$ in our mouse model. Perihematomal edema includes cytotoxic edema due largely to apoptotic and ferroptotic cell death, and vasogenic edema resulting mainly from disruption of the blood-brain barrier [64]. ICH stimulates microglia and astrocytes, leading to production of inflammatory IL-1 $\beta$ and IL-6, which injure endothelial cells. MMP-9 is up-regulated, and it degrades the vascular matrix; while $\mathrm{ZO}-1$ is down-regulated, weakening tight junctions [65]. All these changes compromise the integrity of the bloodbrain barrier. At the same time, IL- $1 \beta$ causes endothelial cells to up-regulate adhesion molecules such as ICAM-1, VCAM-1, and E-selectin, which promote leukocyte margination and adhesion to the endothelial luminal surface, releasing proteases and cytokines and breaking the blood-brain barrier "from the outside" [66]. In our study, DPM significantly reduced the activation of microglia and astrocytes; inhibited neutrophil infiltration; down-regulated IL- $1 \beta$, IL- 6, TNF- $\alpha$ and MMP-9; and up-regulated ZO-1.

Following ICH, cytotoxic edema is accompanied by different forms of death of glial cells and neurons such as apoptosis and ferroptosis [67]. Previous studies have suggested that apoptosis is the main mechanism of early tissue injury in the region surrounding the hematoma after ICH [68]. Many factors induce cell apoptosis after $\mathrm{ICH}$, such as free radical cascade reactions, inflammation, cytokine stimulation, and induction of thrombin and blood components. In the present study, DPM decreased ROS levels in vitro, thereby alleviating oxidative stress and stopping cascade reactions. Consistent with previous findings, we found that $\mathrm{ICH}$ decreased the ratio of $\mathrm{Bcl}-2 / \mathrm{Bax}$ in brain tissues, which was reversed by DRC and DPM, corresponding to less apoptosis of neurons. Since ferroptosis is regarded as one of the main causes of secondary brain injury, reducing it further improves neurological functional outcomes following $\mathrm{ICH}$. We observed that PM and DPM also significantly attenuated ferroptosis of neurons after $\mathrm{ICH}$.

Ferroptosis is a form of regulated cell death characterized by the iron-dependent accumulation of lipid hydroperoxides to lethal cellular levels [69]. GPX4 converts potentially toxic lipid hydroperoxides (L-OOH) to non-toxic lipid alcohols (L-OH). Free iron inhibits the regeneration of glutathione via the Fenton reaction, which inactivates GPX4 and ferroptosis [70]. In the present study, we found that both PM and DPM significantly preserved GPX4, probably by chelating metal ions such as $\mathrm{Cu}^{2+}$ and $\mathrm{Fe}^{2+}$, thereby alleviating 
ferroptosis. Therefore, by simultaneously preventing apoptosis and ferroptosis, DPM may alleviate both early and secondary brain injury for $\mathrm{ICH}$.

\section{Conclusion}

Our studies in a mouse model suggest that DPM can protect against $\mathrm{ICH}$-induced disruption of the $\mathrm{BBB}$, brain edema and neurological deficits. The formulation appears to work by inhibiting $\mathrm{ICH}$-induced activation of neuroglia, infiltration by neutrophils, production of pro-inflammatory factors (IL- $1 \beta$, IL- 6, TNF- $\alpha$ ) and MMP-9, and down-regulation of the tight junction protein ZO-1. The ability of DPM to target both apoptosis and ferroptosis as well as weaken the pro-inflammatory state after ICH may make it an effective therapy.

\section{Supplementary information}

Supplementary information accompanies this paper at https://doi. org/10.1186/s12951-020-00616-3.

Additional file 1. Additional figures.

\section{Acknowledgements}

The authors thank the Department of Radiology at the Affiliated Hospital of Guilin Medical University and the Analytical Centre at Guilin Medical University.

\section{Authors' contributions}

JM led the research. ML carried out biological experiments. LW synthesized and characterized nanomaterials. KW performed drug loading and release experiments. GL performed MRI and analyzed data. XF executed statistical analysis. JM and WC wrote the paper, which LL reviewed. All authors read and approved the final manuscript.

\section{Funding}

This work was supported by the National Natural Science Foundation of China (81860629), Major Project of Guangxi Science and Technology Department (AA17292001), the Open Funds of the Guangxi Key Laboratory of Tumor Immunology and Microenvironmental Regulation (2018KF003), Project for Promoting Scientific Research Ability of Staff in The Affiliated Hospital of Guilin Medical University (2018glmcy034) and State Key Laboratory for Chemistry and Molecular Engineering of Medicinal Resources (Guangxi Normal University) (CMEMR2018-B06).

\section{Availability of data and materials \\ Data and materials are available upon request. \\ The raw/processed data required to reproduce these findings cannot be shared at this time as the data also forms part of an ongoing study.}

\section{Ethics approval and consent to participate}

Animal protocols were approved by the Ethics Committee of Guilin Medical University (License No. YXLL-2017-167), and they complied with the Principles of Laboratory Animal Care (People's Republic of China).

\section{Consent for publication}

All authors concur with the submission and publication of this paper.

\section{Competing interests}

The authors declare that they have no competing interests.

\section{Author details}

${ }^{1}$ Clinical Research Center for Neurological Diseases of Guangxi Province, The Affiliated Hospital of Guilin Medical University, Guilin 541001, China.
${ }^{2}$ School of Pharmacy, Guilin Medical University, Guilin 541001, China. ${ }^{3}$ School of Chemistry, University of New South Wales Sydney, Kensington, NSW 2052, Australia. ${ }^{4}$ Division of Pharmacy, School of Allied Health, University of Western Australia, Perth, WA 6009, Australia. ${ }^{5}$ Department of Radiology, Affiliated Hospital of Jilin Medical University, Jilin 132013, China. ${ }^{6}$ Department of Pharmacy, The Affiliated Hospital of Guilin Medical University, Guilin 541001, China.

Received: 19 November 2019 Accepted: 9 April 2020

Published online: 19 April 2020

\section{References}

1. Toyoda K, Koga M, Yamamoto H, et al. Clinical outcomes depending on acute blood pressure after cerebral hemorrhage. Ann Neurol. 2019:85:105-13.

2. Tsivgoulis G, Wilson D, Katsanos AH, et al. Neuroimaging and clinical outcomes of oral anticoagulant-associated intracerebral hemorrhage. Ann Neurol. 2018:84:694-704.

3. Kumar S, Adjei IM, Brown SB, et al. Manganese dioxide nanoparticles protect cartilage from inflammation-induced oxidative stress. Biomaterials. 2019:224:119467.

4. Xie J, Shen Z, Anraku Y, et al. Nanomaterial-based blood-brain-barrier (BBB) crossing strategies. Biomaterials. 2019:224:119491.

5. Zhu H, Dai C, He L, et al. Iron(II)Polypyridyl complexes as antiglioblastoma agents to overcome the blood-brain barrier and inhibit cell proliferation by regulating p53 and 4E-BP1 Pathways. Front Pharmacol. 2019;10:946.

6. Zhang ZY, Jiang M, Fang J, et al. Enhanced therapeutic potential of nanocurcumin against subarachnoid hemorrhage-induced blood-brain barrier disruption through inhibition of inflammatory response and oxidative stress. Mol Neurobiol. 2017:54:1-14.

7. Qiao B, Wang H, Wang $\mathrm{C}$, et al. Dauricine negatively regulates lipopolysaccharide- or cecal ligation and puncture-induced inflammatory response via NF-kappaB inactivation. Arch Biochem Biophys. 2019;666:99-106.

8. Pu Z, Ma S, Wang $L$, et al. Amyloid-beta degradation and neuroprotection of dauricine mediated by unfolded protein response in a caenorhabditis elegans model of Alzheimer's disease. Neuroscience. 2018;392:25-37.

9. Dong PL, Han H, Zhang TY, et al. Pglycoprotein inhibition increases the transport of dauricine across the blood-brain barrier. Mol Med Rep. 2014;9:985-8.

10. Mo J, Wang L, Huang $X$, et al. Multifunctional nanoparticles for co-delivery of paclitaxel and carboplatin against ovarian cancer by inactivating the JMJD3-HER2 axis. Nanoscale. 2017;9:13142-52.

11. Yuan ZX, Mo J, Zhao G, et al. Targeting strategies for renal cell carcinoma: from renal cancer cells to renal cancer stem cells. Front Pharmacol. 2016:7:423.

12. Wei $L$, Yang $Y$, Shi $K$, et al. Preparation and characterization of loperamideloaded dynasan 114 solid lipid nanoparticles for increased oral absorption in the treatment of diarrhea. Front Pharmacol. 2016;7:332.

13. Wei L, Wang C, Chen X, et al. Dual-responsive, methotrexate-loaded, ascorbic acid-derived micelles exert anti-tumor and anti-metastatic effects by inhibiting NF-kappaB signaling in an orthotopic mouse model of human choriocarcinoma. Theranostics. 2019:9:4354-74.

14. Mo JX, Shi SJ, Zhang Q, et al. Synthesis, transport and mechanism of a type I prodrug: L-carnitine ester of prednisolone. Mol Pharm. 2011;8:1629-40.

15. Mo J, Lim LY, Zhang ZR. L-Carnitine ester of prednisolone: pharmacokinetic and pharmacodynamic evaluation of a type I prodrug. Int J Pharm. 2014:475:123-9.

16. Mo J, Eggers PK, Yuan ZX, et al. Paclitaxel-loaded phosphonated calixarene nanovesicles as a modular drug delivery platform. Sci Rep. 2016;6:23489

17. Mo J, Eggers PK, Raston CL, Lim LY. Development and validation of a LC/ TOF MS method for the determination of carboplatin and paclitaxel in nanovesicles. Anal Bioanal Chem. 2014;406:2659-67.

18. Mo J, Eggers PK, Chen X, et al. Shear induced carboplatin binding within the cavity of a phospholipid mimic for increased anticancer efficacy. Sci Rep. 2015:5:10414.

19. Ming Y, Li Y, Xing H, et al. Circulating tumor cells: from theory to nanotechnology-based detection. Front Pharmacol. 2017:8:35. 
20. Yan J, Zuo G, Sherchan P, et al. CCR1 activation promotes neuroinflammation through CCR1/TPR1/ERK1/2 signaling pathway after intracerebral hemorrhage in mice. Neurotherapeutics. 2020;. https://doi.org/10.1007/ s13311-019-00821-5.

21. Qu X, Wang N, Chen W, et al. RNF34 overexpression exacerbates neurological deficits and brain injury in a mouse model of intracerebral hemorrhage by potentiating mitochondrial dysfunction-mediated oxidative stress. Sci Rep. 2019;9:16296.

22. Wu X, Fu S, Liu Y, et al. NDP-MSH binding melanocortin-1 receptor ameliorates neuroinflammation and BBB disruption through CREB/Nr4a1/NFkappaB pathway after intracerebral hemorrhage in mice. J Neuroinflamm. 2019;16:192.

23. Xiao Y, Li G, Chen Y, et al. Milk fat globule-epidermal growth factor-8 pretreatment attenuates apoptosis and inflammation via the integrin-beta3 pathway after surgical brain injury in rats. Front Neurol. 2018;9:96.

24. Xiong L, Sun L, Liu S, et al. The protective roles of urinary trypsin inhibitor in brain injury following fat embolism syndrome in a rat model. Cell Transpl. 2019;28:704-12.

25. Zhang H, Wang Y, He Z. Glycine-Histidine-Lysine (GHK) alleviates neuronal apoptosis due to intracerebral hemorrhage via the miR-339-5p/ VEGFA pathway. Front Neurosci. 2018;12:644.

26. Meng Z, Zhao T, Zhou K, et al. A20 ameliorates intracerebral hemorrhageinduced inflammatory injury by regulating TRAF6 polyubiquitination. J Immunol. 2017;198:820-31.

27. Yamauchi S, Mano S, Oikawa K, et al. Autophagy controls reactive oxygen species homeostasis in guard cells that is essential for stomatal opening. Proc Natl Acad Sci USA. 2019;116:19187-92.

28. Wang YQ, Chang SY, Wu Q, et al. The protective role of mitochondrial ferritin on erastin-induced ferroptosis. Front Aging Neurosci. 2016;8:308.

29. Slaughter KB, Meyer EG, Bambhroliya AB, et al. Direct assessment of health utilities using the standard gamble among patients with primary intracerebral hemorrhage. Circulation. 2019;12:e005606.

30. Silverman A, Kodali S, Strander S, et al. Deviation from personalized blood pressure targets is associated with worse outcome after subarachnoid hemorrhage. Stroke. 2019;50:2729-37.

31. Ouyang Y, Li D, Wang H, et al. MiR-21-5p/dual-specificity phosphatase 8 signalling mediates the anti-inflammatory effect of haem oxygenase- 1 in aged intracerebral haemorrhage rats. Aging Cell. 2019;2019:e13022.

32. Raposo N, Planton M, Payoux P, et al. Enlarged perivascular spaces and florbetapir uptake in patients with intracerebral hemorrhage. Eur J Nuclear Med Mol Imag. 2019;46:2339-47.

33. Calviere L, Viguier A, Patsoura S, et al. Risk of intracerebral hemorrhage and mortality after convexity subarachnoid hemorrhage in cerebral amyloid angiopathy. Stroke. 2019;50:2562-4.

34. Wu CH, Chen CC, Hung TH, et al. Activation of TrkB/Akt signaling by a TrkB receptor agonist improves long-term histological and functional outcomes in experimental intracerebral hemorrhage. J Biomed Sci. 2019;26:53.

35. Zheng W, Matei N, Pang J, et al. Delayed recanalization at 3 days after permanent MCAO attenuates neuronal apoptosis through FGF21/FGFR1/ PI3K/Caspase-3 pathway in rats. Exp Neurol. 2019;320:113007.

36. Ma C, Gurol ME, Huang Z, et al. Low-density lipoprotein cholesterol and risk of intracerebral hemorrhage: a prospective study. Neurology. 2019;93:e445-57.

37. Zhao S, Liu Z, Yu Z, et al. BIO alleviates inflammation through inhibition of GSK-3beta in a rat model of intracerebral hemorrhage. J Neurosurg. 2019;2019:1-9.

38. Chen AQ, Fang Z, Chen XL, et al. Microglia-derived TNF-alpha mediates endothelial necroptosis aggravating blood brain-barrier disruption after ischemic stroke. Cell Death Dis. 2019;10:487.

39. Divani AA, Liu X, Di Napoli M, et al. Blood pressure variability predicts poor in-hospital outcome in spontaneous intracerebral hemorrhage. Stroke. 2019;50:2023-9.

40. Zhu Q, Gong Y, Guo T, et al. Thermo-sensitive keratin hydrogel against iron-induced brain injury after experimental intracerebral hemorrhage. Int J Pharm. 2019:566:342-51.

41. Ding Y, Flores J, Klebe D, et al. Annexin A1 attenuates neuroinflammation through FPR2/p38/COX-2 pathway after intracerebral hemorrhage in male mice. J Neurosci Res. 2019;98(1):168-78.

42. Xu W, LiT, Gao L, et al. Sodium benzoate attenuates secondary brain injury by inhibiting neuronal apoptosis and reducing mitochondria-mediated oxidative stress in a rat model of intracerebral hemorrhage: possible involvement of DJ-1/Akt/IKK/NFkappaB Pathway. Front Mol Neurosci. 2019;12:105.

43. Gai C, Yu M, Li Z, et al. Acetaminophen sensitizing erastin-induced ferroptosis via modulation of $\mathrm{Nrf2/heme} \mathrm{oxygenase-1} \mathrm{signaling} \mathrm{pathway} \mathrm{in}$ non-small-cell lung cancer. J Cell Physiol. 2019;235(4):3329-39.

44. Lang X, Green MD, Wang W, et al. Radiotherapy and immunotherapy promote tumoral lipid oxidation and ferroptosis via synergistic repression of SLC7A11. Cancer Discov. 2019;9(12):1673-85.

45. Xia M, Chen W, Wang J, et al. TRPA1 activation-induced myelin degradation plays a key role in motor dysfunction after intracerebral hemorrhage. Front Mol Neurosci. 2019;12:98.

46. Luo $Q, L i$ D, Bao B, et al. NEMO-binding domain peptides alleviate perihematomal inflammation injury after experimental intracerebral hemorrhage. Neuroscience. 2019;409:43-57.

47. Ding D, Chen CJ, Starke RM, et al. Risk of brain arteriovenous malformation hemorrhage before and after stereotactic radiosurgery. Stroke. 2019:50:1384-91.

48. Chen TY, Lin CL, Wang LF, et al. Targeting GPER1 to suppress autophagy as a male-specific therapeutic strategy for iron-induced striatal injury. Sci Rep. 2019;9:6661.

49. Han Y, Seyfried D, Meng Y, et al. Multipotent mesenchymal stromal cell-derived exosomes improve functional recovery after experimental intracerebral hemorrhage in the rat. J Neurosurg. 2018;2018:1-11.

50. Jiang C, Zou X, Zhu R, et al. The correlation between accumulation of amyloid beta with enhanced neuroinflammation and cognitive impairment after intraventricular hemorrhage. J Neurosurg. 2018;2018:1-10.

51. Yu H, Geng WC, Zheng Z, et al. Facile fluorescence monitoring of gut microbial metabolite trimethylamine $\mathrm{N}$-oxide via molecular recognition of guanidinium-modified calixarene. Theranostics. 2019;9:4624-32.

52. Renziehausen $A$, Tsiailanis AD, Perryman $R$, et al. Encapsulation of temozolomide in a calixarene nanocapsule improves its stability and enhances its therapeutic efficacy against glioblastoma. Mol Cancer Ther. 2019;18:1497-505.

53. Wang Y, Luo Y, Zhao Q, et al. An enzyme-responsive nanogel carrier based on PAMAM dendrimers for drug delivery. ACS Appl Mater Interfaces. 2016;8:19899-906.

54. Chen W, Li L, Zhang X, et al. Curcumin: a calixarene derivative micelle potentiates anti-breast cancer stem cells effects in xenografted, triplenegative breast cancer mouse models. Drug Deliv. 2017;24:1470-81.

55. He L, Gu J, Lim LY, et al. Nanomedicine-mediated therapies to target breast cancer stem cells. Front Pharmacol. 2016;7:313.

56. Li B, Li Q, Mo J, Dai H. Drug-loaded polymeric nanoparticles for cancer stem cell targeting. Front Pharmacol. 2017;8:51.

57. Li H, Yan L, Tang EKY, et al. Synthesis of TPGS/curcumin nanoparticles by thin-film hydration and evaluation of their anti-colon cancer efficacy in vitro and in vivo. Front Pharmacol. 2019;10:769.

58. Imai T, Iwata S, Hirayama T, et al. Intracellular Fe(2+) accumulation in endothelial cells and pericytes induces blood-brain barrier dysfunction in secondary brain injury after brain hemorrhage. Sci Rep. 2019;9:6228.

59. Liu H, Xu H, Jiang Y, et al. Preparation, characterization, in vivo pharmacokinetics, and biodistribution of polymeric micellar dimethoxycurcumin for tumor targeting. Int J Nanomed. 2015;10:6395-410.

60. Dhand C, Prabhakaran MP, Beuerman RW, et al. Role of size of drug delivery carriers for pulmonary and intravenous administration with emphasis on cancer therapeutics and lung-targeted drug delivery. RSC Adv. 2014;4:32673-89.

61. Jiao $X, \mathrm{He}$, LiY, et al. The role of circulating tight junction proteins in evaluating blood brain barrier disruption following intracranial hemorrhage. Dis Mark. 2015;2015:860120.

62. Ohtsuki S, Yamaguchi H, Asashima T, Terasaki T. Establishing a method to isolate rat brain capillary endothelial cells by magnetic cell sorting and dominant mRNA expression of multidrug resistance-associated protein 1 and 4 in highly purified rat brain capillary endothelial cells. Pharm Res. 2007;24:688-94.

63. Bourassa P, Alata W, Tremblay C, et al. Transferrin receptor-mediated uptake at the blood-brain barrier is not impaired by Alzheimer's disease neuropathology. Mol Pharm. 2019;16:583-94.

64. Chen J, Hu J, Liu H, et al. FGF21 protects the blood-brain barrier by upregulating PPARgamma via FGFR1/beta-klotho after traumatic brain injury. J Neurotrauma. 2018;35:2091-103. 
65. Tang $\mathrm{Y}$, Le W. Differential roles of $\mathrm{M} 1$ and $\mathrm{M} 2$ microglia in neurodegenerative diseases. Mol Neurobiol. 2016;53:1181-94.

66. Fang Y, Tian Y, Huang Q, et al. Deficiency of TREK-1 potassium channel exacerbates blood-brain barrier damage and neuroinflammation after intracerebral hemorrhage in mice. J Neuroinflamm. 2019;16:96.

67. Karuppagounder SS, Alin L, Chen Y, et al. N-acetylcysteine targets 5 lipoxygenase-derived, toxic lipids and can synergize with prostaglandin E2 to inhibit ferroptosis and improve outcomes following hemorrhagic stroke in mice. Ann Neurol. 2018:84:854-72.

68. Parry-Jones AR, Sammut-Powell C, Paroutoglou K, et al. An intracerebral hemorrhage care bundle is associated with lower case fatality. Ann Neurol. 2019:86:495-503.
69. Dodo K, Kuboki E, Shimizu T, et al. Development of a water-soluble indolylmaleimide derivative IM-93 showing dual inhibition of ferroptosis and NETosis. ACS Med Chem Lett. 2019:10:1272-8.

70. Bi J, Yang $\mathrm{S}$, Li L, et al. Metadherin enhances vulnerability of cancer cells to ferroptosis. Cell Death Dis. 2019;10:682.

\section{Publisher's Note}

Springer Nature remains neutral with regard to jurisdictional claims in published maps and institutional affiliations.
Ready to submit your research? Choose BMC and benefit from

- fast, convenient online submission

- thorough peer review by experienced researchers in your field

- rapid publication on acceptance

- support for research data, including large and complex data types

- gold Open Access which fosters wider collaboration and increased citations

- maximum visibility for your research: over 100M website views per year

At BMC, research is always in progress.

Learn more biomedcentral.com/submissions 